\title{
A Mathematical Model for Germinal Centre Kinetics and Affinity Maturation
}

\author{
Dagmar Iber $* \dagger$ And Philip K. Maini $\dagger$ \\ $\dagger$ Centre for Mathematical Biology, Mathematical Institute, 24-29 St Giles, Oxford OX1 3LB, U.K.
}

(Received on 7 December 2001, Accepted in revised form on 13 May 2002)

\begin{abstract}
We present a mathematical model which reproduces experimental data on the germinal centre (GC) kinetics of the primed primary immune response and on affinity maturation observed during the reaction. We show that antigen masking by antibodies which are produced by emerging plasma cells can drive affinity maturation and provide a feedback mechanism by which the reaction is stable against variations in the initial antigen amount over several orders of magnitude. This provides a possible answer to the long-standing question of the role of antigen reduction in driving affinity maturation. By comparing model predictions with experimental results, we propose that the selection probability of centrocytes and the recycling probability of selected centrocytes are not constant but vary during the GC reaction with respect to time. It is shown that the efficiency of affinity maturation is highest if clones with an affinity for the antigen well above the average affinity in the GC leave the GC for either the memory or plasma cell pool. It is further shown that termination of somatic hypermutation several days before the end of the germinal centre reaction is beneficial for affinity maturation. The impact on affinity maturation of simultaneous initiation of memory cell formation and somatic hypermutation vs. delayed initiation of memory cell formation is discussed.
\end{abstract}

(C) 2002 Elsevier Science Ltd. All rights reserved.

\section{Introduction}

Affinity maturation refers to the increase in affinity of the antibodies for antigen produced during the course of an immune response (Janeway \& Travers, 1997). This is achieved by mutation of the genes encoding for the antibody and subsequent selection of those B cells which express B cell receptors with the highest affinity for antigen (MacLennan et al., 2000). How mutation and selection are regulated is currently

\footnotetext{
*Corresponding author. Tel.: +44-1223-402250; fax: $+44-1223-412178$

$\dagger$ Present address: MRC-LMB, Hills Road, Cambridge CB2 2QH, U.K.

E-mail addresses: di205@cam.ac.uk (D. Iber),maini@ maths.ox.ac.uk (P.K. Maini).
}

unknown. Both processes are believed to take place in the germinal centres of secondary lymphoid organs (Berek et al., 1991; Jacob et al., 1991).

Upon immunization (infection) the antigen is concentrated in the secondary lymphoid organs where a small subset of all $B$ cells recognize it (Goodnow, 1997). After successful presentations of antigen fragments to T cells, B cells enter the blast state (MacLennan et al., 2000). A subset of the antigen-specific $\mathrm{B}$ and $\mathrm{T}$ cells enter the follicle and continue dividing about 4 times a day (Liu et al., 1991). After about 3 days B blasts differentiate into centroblasts which still divide every 6-7 hr and after 4 days a spatial reorganization can be observed. It has been shown that 
centrocytes are the progenitors of centroblasts (Liu et al., 1991). Centrocytes express B cell receptors on their surface and are believed to be subject to selection by antigen in the form that they need to bind, internalize and present antigen to T cells (Lanzavecchia, 1985) in order not to undergo apoptosis (MacLennan, 1994). Those centrocytes that successfully interact with $\mathrm{T}$ cells can either differentiate into memory or plasma cells (MacLennan, 1994). There is substantial experimental evidence for a third differentiation pathway back into the centroblast state (de Vinuesa et al., 2000; Han et al., 1995; Liu et al., 1991).

Soon after the first differentiation of centroblasts into centrocytes, somatic hypermutation starts to act on centroblasts and leads to a change in the affinity of the $\mathrm{B}$ cell receptors for antigen. About half of the mutations (53\%) have been estimated to be of a silent nature not leading to any change in affinity (Shannon \& Mehr, 1999). About 28\% have been estimated to lead to B cell death which is in good agreement with the apoptotic cells observed in the dark zone where centroblasts proliferate (Hardie et al., 1993; Liu et al., 1991). Nineteen percent of all mutations have been estimated to have an effect on affinity. This effect can either be of an improving nature and drive affinity maturation, or of worsening or specificity changing character which may even lead to the formation of autoreactive clones. Selection is therefore clearly needed. It is currently unclear how exactly the read-out may work. Several studies support the concept that antigen is presented on the surface of follicular dendritic cells (FDCs) (Liu et al., 1989) and that this membrane presentation may lower the affinity threshold (Batista \& Neuberger, 2000) and the required amount of deposited antigen (Batista et al., 2001) such that early clones of low affinity can also successfully extract and present antigen. The threshold needs to be dynamic with respect to time in order to drive affinity maturation. This dynamic nature may derive from increasing competition between centrocytes for free antigen which is probably reduced by antigen extraction (Batista et al., 2001) and masking through antibodies produced by early plasma cells (Ridderstad \& Tarlinton, 1998; Tarlinton \& Smith, 2000). In Table 1, we summarize the key stages of the primed primary immune response.

How activation of $B$ cells by antigen and $T$ cells determines their fate, for example, whether they differentiate into either centroblasts, memory or plasma cells, is currently unknown and there is no experimental system available to investigate this. A mathematical model is therefore helpful for investigating hypotheses and checking their compatibility with available data.

Before investigating affinity maturation we first need to construct a model of the germinal centre (GC) kinetics which reproduces all available data on the relevant population dynamics that are related to the GC reaction. Into this model the model of affinity maturation can then

TABLE 1

GC kinetics in the primed primary immune response

\begin{tabular}{|c|c|c|}
\hline Days & Action & References \\
\hline 1 & Immunization & \\
\hline First 3 & B blast proliferation with 1 division within $6-7 \mathrm{hr}$ & Liu et al., (1991) \\
\hline 3 & First apoptosis (no memory cells yet) & MacLennan (pers. comm., 2001) \\
\hline \multirow[t]{3}{*}{$3-4$} & $\begin{array}{l}\text { Differentiation of B blasts into centroblasts } \\
\text { Onset of somatic hypermutation }\end{array}$ & Liu et al. (1991) \\
\hline & (at $96 \mathrm{hr}$ already two generations of mutations) & MacLennan (pers. comm., 2001) \\
\hline & Start of memory cell formation & Liu et al. (1991) and MacLennan (pers. comm., 2001) \\
\hline 4 & $\begin{array}{l}\text { GC formation: centroblast (dark zone) and } \\
\text { centrocytes (light zone) }\end{array}$ & $\begin{array}{l}\text { Jacob et al. (1991), Jacob \& Kelsoe (1992) and } \\
\text { Liu et al. (1991) }\end{array}$ \\
\hline & GC biggest now & Liu et al. (1991) \\
\hline 5 & $\begin{array}{l}\text { Mutated plasma cells can be found (may have } \\
\text { already appeared earlier) }\end{array}$ & MacLennan (pers. comm., 2001) \\
\hline 21 & $\mathrm{GC}$ reaction finishes & $\begin{array}{l}\text { Jacob et al. (1991), Jacob \& Kelsoe (1992) and } \\
\text { Liu et al. (1991) }\end{array}$ \\
\hline
\end{tabular}


be embedded. Previous mathematical models of the process were very complex and unable to reproduce all data on the germinal centre reaction. An important condition for being able to construct a good model is the availability of a large body of reliable experimental data. The model presented below is therefore constructed for the primed, instead of for the true, primary immune response, since more (and also more accurate) data are available for the former. In the primed primary immune response an animal is first immunized with a precipitated carrier protein that activates $T$ cells. A month later the animal is again immunized with the carrier protein but this time it is conjugated to a hapten. A hapten is an agent that is too small to raise an immune response but, when conjugated to a carrier protein, it is recognized by the immune system and raises an immune response. It is important to note that the hapten-carrier protein complex can be injected in soluble form. This leads to a faster clearance of the antigen from the body and a sharper response which allows a more accurate description of the process and therefore yields better data. The problem of modelling the true primary immune response already begins with deciding on the exact time of onset of the immune response, since this is smeared over a time span of several days because the antigen needs to be injected in precipitated form and dissolves slowly. A drawback of modelling the primed instead of the true primary immune response is that due to the priming, $\mathrm{T}$ cell help is non-limiting and the immune response against the hapten is faster and stronger than in the unprimed case. This is an unfortunate side effect since the mechanisms of the two responses may therefore be different and an understanding of the artificial primed primary immune response does not directly imply an understanding of the true primary immune response. Many features will however be similar although the kinetics differ (Liu et al., 1991) and an understanding of the primed primary immune response may therefore facilitate an understanding of the true primary immune response. In the following, a simplistic mathematical model is devised with which all available data can be reproduced and predictions can be made concerning selection strategies and the regulation of the differentiation of selected centrocytes into either memory, plasma cells or centroblasts.

\section{The Basic Model}

Calculations based on the emergence of antigen-specific B cells led to the suggestion that follicles are seeded during the first hours of infection (immunization in experiments) and that each follicle soon becomes dominated by five (or fewer) clones (Jacob et al., 1991; Kroese et al., 1987; Liu et al., 1991). In the model it is therefore assumed that the GCs are seeded by three clones which give rise to $12288 \mathrm{~B}$ blasts within 3 days if we use the proliferation rate $\rho=$ $\frac{24}{6.5} \ln 2$ day $^{-1}$ for centroblasts determined by Liu and co-workers (Liu et al., 1991). At day 3, B blasts differentiate into centroblasts and the main GC reaction starts. The model, presented schematically in Fig. 1, takes the form

$$
\begin{gathered}
\frac{\mathrm{d} B}{\mathrm{~d} t}=p_{r} \rho C_{s}-\rho B, \\
\frac{\mathrm{d} C}{\mathrm{~d} t}=2 \rho m B-\mu C \quad \text { with } \mu=d s+\delta_{c}(1-s), \\
\frac{\mathrm{d} C_{s}}{\mathrm{~d} t}=d s C-\rho C_{s}, \\
\frac{\mathrm{d} M}{\mathrm{~d} t}=\left(1-p_{r}\right) \rho C_{s}
\end{gathered}
$$

and is derived in the following way. There is no experimental work on the regulative mechanism for differentiation of centroblasts into centrocytes. In a previous model, Kesmir and de Boer (1999) assumed that centroblasts differentiate into centrocytes after a set number of generations $n$, which is larger than 4 . One can show that other mechanisms, such as time-dependent or cell-density-dependent centroblast-centrocyte conversion, cannot satisfy all experimental data on population dynamics of certain B cell subpopulations. In the case of a cell-densitydependent conversion mechanism, we find a non-trivial stable steady state which is rapidly attained and prohibits termination of the response. This problem was encountered before by Oprea and Perelson who got around it by 


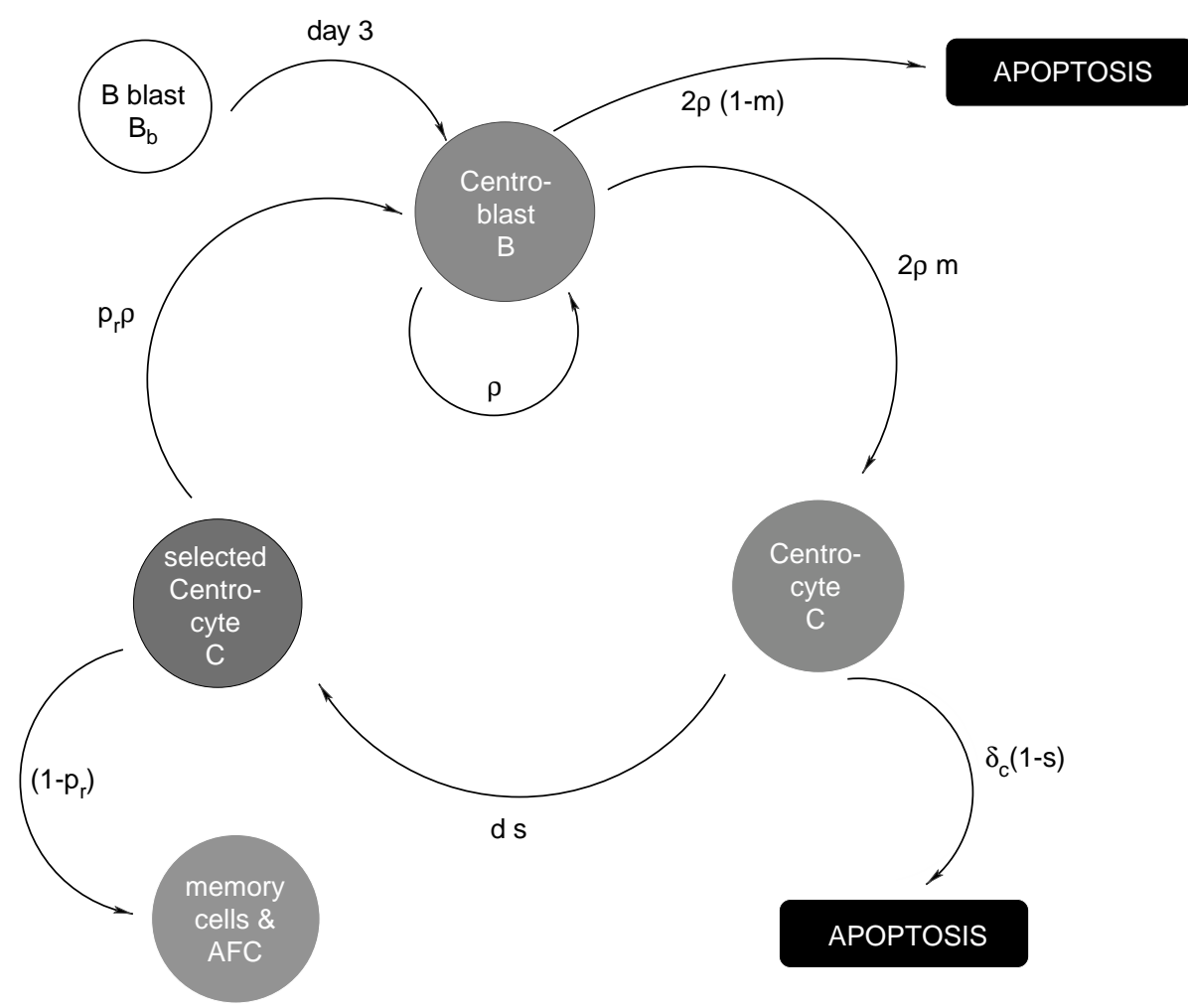

FIG. 1. Scheme of the model for the primed primary immune response. Abbreviations are explained in Table 2.

introducing a sudden emigration of GC cells at the time when biologists measured the dissolution of germinal centres (Oprea \& Perelson, 1997). This is, however, a phenomenological approach that cannot explain the underlying mechanism. The steady state can be circumvented in the time-dependent conversion model but only at the expense of losing another GC feature: the model then does not reproduce the sudden loss of GCs when recycling is impaired (de Vinuesa et al., 2000). In this model we therefore assume generation-dependent centroblast differentiation and it will be shown later that agreement with the experimental observations of MacLennan and co-workers (MacLennan et al., 1990) only occurs if we assume that centroblasts already differentiate into centrocytes after one cell division.

The conversion of centroblasts into centrocytes could involve only one or both daughter cells. However, in the former case we would not obtain the observed GC kinetics since the centroblast pool could only increase but not decrease since no net outflux term would be included and the GC reaction would never terminate. Hence, in the model it is assumed that centroblast division directly leads to differentiation of both daughter cells into centrocytes and we therefore have the term $-\rho B$ in eqn (1). Due to somatic hypermutation only the proportion $m$ survives the cell division and differentiates into centrocytes while the remaining proportion $(1-m)$ of dividing centroblasts is assumed to die at the same rate as the surviving part differentiates. The death rate of centroblasts may well be smaller than the conversion rate. Such a possible difference cannot, however, be expected to change the modelled kinetics substantially if we assume that centroblasts, once committed to apoptosis, cannot take part in the normal cell division process. A smaller death rate would therefore only lead to a slower decay of the total cell number. The surviving centroblast proportion gives rise to the term $2 \rho m B$ in eqn (2). The factor 2 comes in since one centroblast gives rise to two centrocytes and division of centroblasts is assumed to lead directly to differentiation into centrocytes. 
TABLE 2

Parameters for the minimal model

\begin{tabular}{|c|c|c|}
\hline$B$ & Centroblasts & \\
\hline$C$ & Centrocytes & \\
\hline$C_{s}$ & Centrocytes that have been selected & \\
\hline$M$ & Memory cells & \\
\hline$A F C$ & Antibody forming cells & \\
\hline$\rho=\frac{24}{6.5} \ln 2$ & Maximum proliferation rate of centroblast $\left(\mathrm{day}^{-1}\right)$ & Liu et al. (1991) \\
\hline$m=0.72$ & Probability of non-deleterious mutation & Shannon \& Mehr (1999) \\
\hline$d=12 \ln 2$ & $\begin{array}{l}\text { Rate of running through the selection } \\
\text { process }\left(\text { day }^{-1}\right)\end{array}$ & Liu et al. (1991) \\
\hline$\delta_{c}=1.5 \ln 2$ & Death rate of centrocytes $\left(\right.$ day $\left.^{-1}\right)$ & MacLennan et al. (1990) \\
\hline$s \in[0,1]$ & Selection probability of centrocytes & \\
\hline$p_{r} \in[0,1]$ & Probability of recycling & \\
\hline$\kappa=10^{8}$ & $\begin{array}{l}\text { Production rate of high affinity antibodies that are } \\
\text { retained within the GC (antibody/day } \times \text { cell) }\end{array}$ & \\
\hline$k_{\text {on }}=5 \times 10^{-13}$ & $\begin{array}{l}\text { Antibody-antigen effective association rate } \\
\left(\text { day }^{-1} \text { particle }^{-1}\right)\end{array}$ & Batista \& Neuberger (1998) and Neri et al. (1995) \\
\hline
\end{tabular}

Part of the centrocyte population $(s C)$ becomes selected into the pool $C_{s}$ at a rate $d$ (Liu et al., 1991) via interaction with antigen and $\mathrm{T}$ cells (whose dynamics are not included in this first simple model). This generates the term $d s C$ in eqn (3). The other part $((1-s) C)$ undergoes apoptosis with rate $\delta_{c}$ (MacLennan et al., 1990) and the outflux from the centrocyte pool is therefore in total $\left(d s+\delta_{c}(1-s)\right) C$, which is written as $\mu C$ in eqn (2). The selected centrocytes $\left(C_{s}\right)$ can then take either of two routes: they may, with probability $\left(1-p_{r}\right)$, differentiate into memory $(M)$ or antibody-forming plasma cells $(A F C)$ (at this stage we do not differentiate between these two cell types and denote both populations by $M$ ); or they may, with probability $p_{r}$, recycle and replenish the centroblast pool. The time needed to recycle is assumed to be, on average, about $6 \mathrm{hr}$ and therefore we again use $\rho$ as a rate constant, leading to the term $p_{r} \rho C_{s}$ in eqn (1). We further assume that selected centrocytes differentiate at the same rate into memory or plasma cells as they recycle so that the net outflux term from the pool of selected centrocytes is given by $-\rho C_{s}$ in eqn (3). By the same argument, the influx into the memory pool (4) is given by $\left(1-p_{r}\right) \rho C_{s}$. A different efflux rate from the GC would only change the total number of cells fluxing into the memory and plasma cell pool. Both numbers are currently unknown and difficult to measure since both memory and plasma cells emigrate from the follicles and are then difficult to track.
Non-dimensionalization of the model does not lead to any new insight since the form of the equations remains unchanged (that is, there are no small parameters that we can exploit see Table 2 ). We note that the $M$ equation is decoupled from the rest of the system, so we analyze the first three equations of the model system. This system always has a trivial equilibrium point. A nontrivial equilibrium point, exists if

$$
\mu=\mu_{c}=2 p_{r} m s d .
$$

At the equilibrium point, $s$ therefore takes the value

$$
s_{c}=\frac{\delta_{c}}{d\left(2 p_{r} m-1\right)+\delta_{c}} .
$$

Given that the selection factor $s$ can only take values between 0 and 1 in the model, we obtain as a further condition on the denominator

$$
p_{r} m \geqslant 0.5
$$

in order to attain the equilibrium state within the possible parameter range. Due to somatic hypermutation the survival rate $m$ has been calculated to be about 0.72 (Shannon \& Mehr, 1999 ) or 0.75 (Allen et al., 1987). Other sources are more pessimistic and consider $m=0.5$ (Kepler \& Perelson, 1993). For the latter parameter value of $m$ we would need $p_{r}=1$ in order to meet condition (7). $p_{r}$, however, cannot attain value 1 since then no memory cell 
formation would be possible, [eqn (4)]. We therefore have $m>0.5$ as a necessary condition for a non-trivial equilibrium state. For $m \leqslant 0.5$ we have $\mu>\mu_{c}$ for all parameters in the parameter range. Simple analysis shows that the solution blows up for $\mu<\mu_{c}$ while for $\mu>\mu_{c}$ it tends to the trivial equilibrium point. If we use $m=0.72$ on the other hand, we only need $p_{r} \geqslant 0.69$ in order to attain a non-trivial equilibrium state.

\section{Results and Predictions}

\section{TERMINATION OF SOMATIC HYPERMUTATION AT DAY 16 REQUIRES ENHANCEMENT IN THE STRINGENCY OF SELECTION}

Experimental observations show that the overall shape of the GC kinetics during the primed primary immune response (Liu et al., 1991) is characterized by an exponential increase in cell numbers until day 3 or day 4 , when the number of cells reaches about $1-1.5 \times 10^{4}$ (Kuppers et al., 1993; Liu et al., 1991) followed by a halving of cell numbers until day 7 . After day 7 cell numbers decline more slowly until the GC reaction finishes at about day 21 (Liu et al., 1991).

In order to model the decrease in cell numbers we have to determine the set of parameters for which the model attains the trivial equilibrium state. It is generally believed that the decrease in cell population during the GC reaction is mainly due to non-selected centrocytes which are dying (MacLennan et al., 2000). It is further believed that selection is critically dependent on the amount of available antigen, which can be reduced during the reaction by antigen extraction (Batista et al., 2001) and antigen masking through soluble antibody produced by early plasma cells (Ridderstad \& Tarlinton, 1998; Tarlinton \& Smith, 2000). From the latter it follows that the value of the selection factor will only decrease during the GC reaction. The weaker decrease in cell numbers after day 7 must therefore be due to the increase of the other undetermined parameter, the recycling probability $p_{r}$. This assumption can be justified by the experimental result that early clones of high affinity have been observed to preferentially leave the GC reaction. We will come back to this point later. At this stage it is sufficient to assume that those centrocytes which receive a very strong activation signal compared to the average centrocyte population preferentially leave the GC reaction. Given that $\mathrm{B}$ cell and subsequent $\mathrm{T}$ cell activation (interleukin-2 production) is dependent on the availability of antigen (Batista et al., 2001), and given further that the curve of $\mathrm{T}$ cell activation as a function of antigen density is of sigmoidal shape (Batista et al., 2001), the behaviour can best be modelled by the use of a Hill function. We use

$$
p_{r}=0.6+0.2 \frac{A g_{\text {min }}^{n}}{A g^{n}+A g_{\text {min }}^{n}},
$$

where $A g$ is antigen density, $A g_{\min }=7500$ and $n=50$. The parameters $A g_{\text {min }}$ and $n$ are chosen such that the experimental kinetics can be reproduced.

The amount of unbound antigen decreases during the GC reaction due to antigen consumption and masking by high-affinity soluble antigen. The latter will be included once we have modelled affinity maturation. At this stage we only consider antigen reduction by extraction through centrocytes. The initial number of antigen complexes in the lymph node has been estimated to be $5 \times 10^{8}$ (Tew \& Mandel, 1979) although this number will vary between immune responses (MacLennan, Pers. comm., 2001). Given that every GC consists of about $1.5 \times$ $10^{5}$ lymphoid cells and every spleen about 5-7 $\times 10^{7}$ lymphoid cells, the number of GCs can be estimated to be about $300-500$. So there are at least $10^{6}$ antigen molecules per GC. If we follow the estimate in Kesmir \& de Boer (1999) there are 100 FDCs per GC and therefore on average $10^{4}$ antigen molecules per FDC.

The natural decay of antigen is more or less irrelevant since this natural decay is much slower than all active processes. The antigen decay is therefore probably mainly due to antigen extraction by centrocytes. It is reasonable to assume that the more the extraction, the more the centrocytes interact with antigen and the higher is the antigen density on FDCs, since this may facilitate extraction. Assuming further, for simplicity, a linear influence, we are left with $-u C_{S} A g$ as the term modelling the extraction by 
selected centrocytes, where $A g$ is the antigen density. Given that centrocytes which do not manage to become selected may extract antigen as well, we also need to take this proportion of non-selected centrocytes into account. This cell proportion is taken to be $k\left(C-C_{s}\right)$ and we therefore obtain the second term modelling the antigen dynamics to be $-u k\left(C-C_{S}\right) A g$ yielding in total for the antigen dynamics

$$
\frac{\mathrm{d} A g}{\mathrm{~d} t}=-\left(u k C+u C_{s}(1-k)\right) A g
$$

Since $k$ is not known from experiments we assume, for simplicity, that $k=0$. We can easily change this once experimental data are available but given that $u$ and the initial and final antigen density are unknown it does not seem appropriate to explore $k$ at this stage. There is no evidence in the literature that the antigen consumption rate depends on the quality of BCR:antigen interaction and $u$ is therefore taken to be constant. The exact value of $u=2.5 \times$ $10^{-5}$ particle $\times$ cell $^{-1}$ day $^{-1}$ was chosen with the result in mind that within 30 days about half of the antigen has disappeared from the lymph nodes (Tew \& Mandel, 1979). Hence there is still a lot of antigen available in the lymphoid organs when the GC reaction terminates and lack of antigen is therefore unlikely to be the primary reason for GC termination (as proposed previously, Kesmir \& de Boer, 1999). An alternative model (Meyer-Hermann et al., 2001) proposes that $\mathrm{GC}$ termination is due to emigration of GC $\mathrm{B}$ cells as memory or plasma cells and this is the view that we will take. In the model of affinity maturation we will see how antigen masking can affect selection and emigration which finally leads to GC termination. The results for this model can be seen in the top panel of Fig. 2. The total number of $\mathrm{B}$ cells peaks at day 4 with about $1.2 \times 10^{4} \mathrm{~B}$ cells. This is in good agreement with the experimental results of Liu and co-workers (Liu et al., 1991). MacLennan and co-workers find that centrocytes greatly outnumber centroblasts during the main part of the GC reaction and this is also found in the model results. We conclude from the results shown in Fig. 2 that it is sufficient to assume that the recycling rate can change with respect to time-while the selection factor can be kept constant ( $s=0.28)$ - in order to reproduce the experimental kinetics of the GC reaction.

Jacob and co-workers (Jacob et al., 1993) deduced from their experimental results that either the selection stringency has to increase or somatic hypermutation has to terminate at about day 16. It is possible to enhance selection stringency (reduce $s$ ) in this model and still to reproduce the experimental kinetics (data not shown) but there is nothing that would justify this. If, however, somatic hypermutation terminated at day 16 (as was proposed in Berek et al., 1991) then the somatic hypermutation survival rate would increase from $m=0.72$ to 1 . From eqn (6) we see that $s_{c}$ decreases when $m$ increases. Upon termination of somatic hypermutation the selection rate $s$ therefore has to decrease in order to prevent the GC reaction from growing. As discussed above, the selection rate is believed to depend on the amount of available antigen and the behaviour can therefore best be modelled by again using a Hill function. Thus,

$$
\begin{aligned}
& s=s_{c}-h-f \frac{A g_{\text {min }}^{n}}{A g_{\text {min }}^{n}+A g^{n}} \\
& \text { with } \quad A g_{\text {min }}=5000, \quad n=20 .
\end{aligned}
$$

We see that the selection rate is constant $(s=$ $s_{c}-h$ ) for a large range of antigen densities but at a critical value of antigen availability the selection factor $s$ falls rapidly to a constant value $\left(s=s_{c}-h-f\right)$. Given that the selection factor is especially important in the time regime $t>7$, the value of $s_{c}$ is calculated with the parameters of this time regime $\left(p_{r}=0.8, m=0.72\right)$ and we have $s_{c}=0.45$. The parameter $h$ needs to be introduced since the total $B$ cell number decreases between day 7 and day 16 when antigen consumption is not very important. Therefore, the selection factor needs to be smaller than the equilibrium value of $s$ and in order to reproduce the experimental data we chose $h=0.07$. The factor $f$ is needed in order to keep $s$ in the range $[0,1]$ and we chose $f=0.35$ in order to reproduce the experimental data. The Hill coefficient for the recycling probability given in eqn (8) can be reduced to $n=20$. 

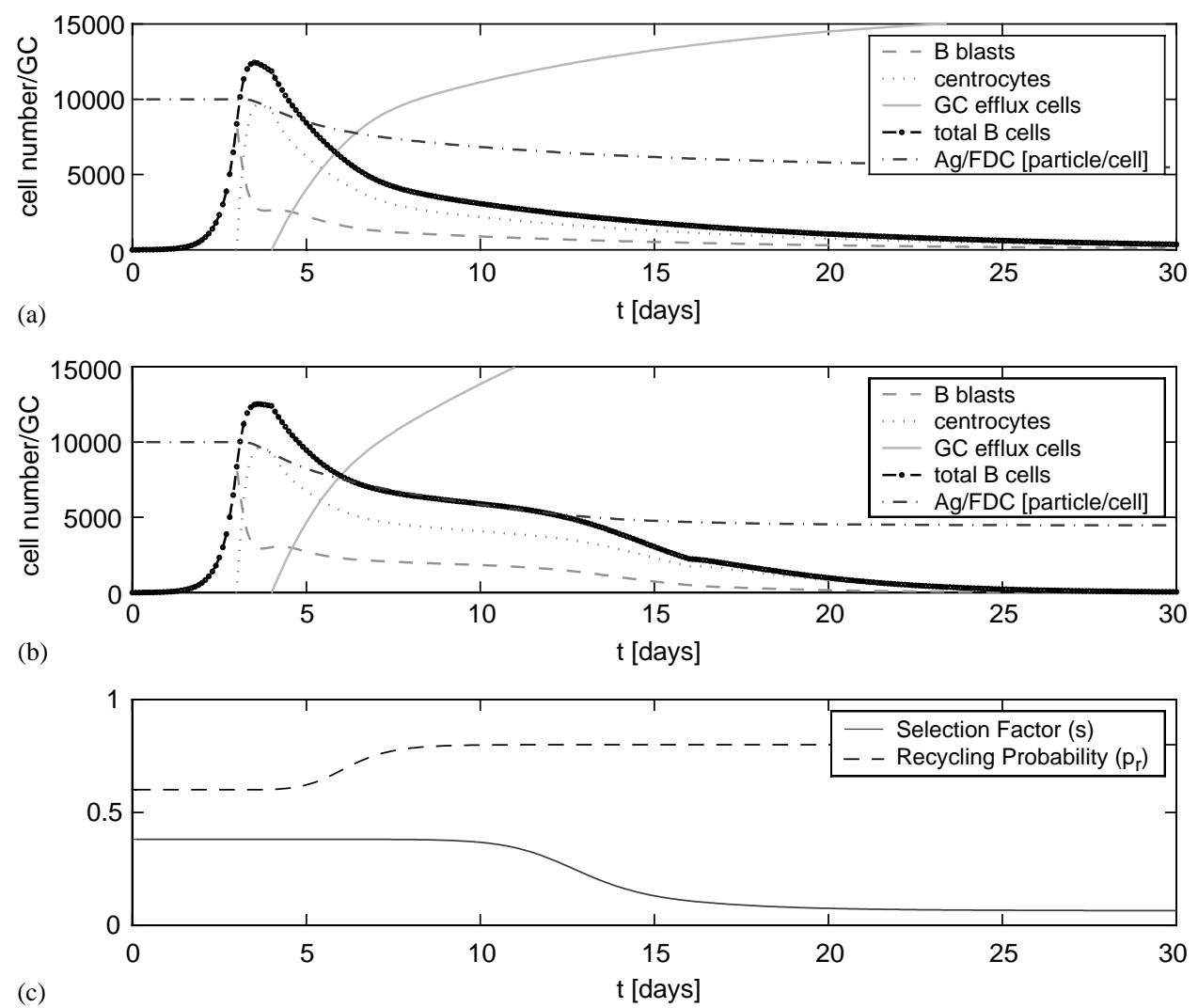

FIG. 2. (a) GC kinetics without termination of somatic hypermutation. The recycling probability is chosen as given in eqn (8). The selection factor $s=0.28$ is chosen constant. (b) GC kinetics with termination of somatic hypermutation at day 16. The final number of GC efflux cells is $2.1 \times 10^{4}$. The recycling probability used is given in eqn $(8)$ with $n=20$, and the selection rate is given in eqn (10). (c) The selection factor $s$ and the recycling probability $p_{r}$ as in (b).

The Hill coefficient $n$ is a measure of the cooperativity of a process and a Hill coefficient of 3 is already considered high (Stryer, 1995). The reason such large values of the Hill coefficient had to be chosen in this model is that the selection rate and the recycling probability need to change rapidly and we see from Fig. 2 that there is no alternative if we want to satisfy the kinetic data. We have therefore to assume that the fall of the selection factor is almost an on/off response where signalling is changed rapidly when the antigen density and affinity reaches a certain threshold. If we understand the rapid decrease in the selection proportion as an effect of a signalling cascade with many amplification steps, a high Hill coefficient, which would not be justifiable for single moleculemolecule interactions, can still be realistic. Such a rapid change, however, makes the model very unstable against small variations in the initial conditions. When we include affinity maturation we will be able to model the selection factor and the recycling probability more mechanistically without having to use Hill functions, thus this problem will not arise.

The middle panel in Fig. 2 shows the results of the model which compare even more closely with the experimental data (Liu et al., 1991) than the results in the top panel, which were obtained for continuous somatic hypermutation and constant selection rate. In the following we will further examine the benefits of termination of somatic hypermutation well before the end of the GC reaction with respect to affinity maturation.

\section{CENTROBLASTS DIFFERENTIATE INTO CENTROCYTES AFTER ONE GENERATION}

After having introduced the basic model for one centroblast generation and having shown 
that it reproduces, reasonably well, kinetic data on the primed primary immune response, we now introduce a multi-generation model and show by comparison with experimental data that a one-generation model agrees best with experimental data. Up to day 3 the general multigeneration model is the same as before, but after day 3 it is given by

$$
\frac{\mathrm{d} B^{0}}{\mathrm{~d} t}=p_{r} \rho C_{s}-\rho B^{0},
$$

$\frac{\mathrm{d} B^{j}}{\mathrm{~d} t}=2 \rho m B^{j-1}-\rho B^{j} \quad$ for $j=1, \ldots, n$,

$$
\frac{\mathrm{d} C}{\mathrm{~d} t}=2 \rho m B^{n}-\mu C \quad \text { with }
$$

$$
\mu=d s+\delta_{c}(1-s)
$$

$$
\begin{aligned}
& \frac{\mathrm{d} C_{s}}{\mathrm{~d} t}=d s C-\rho C_{s}, \\
& \frac{\mathrm{d} M}{\mathrm{~d} t}=\left(1-p_{r}\right) \rho C_{s},
\end{aligned}
$$

where the upper indices indicate the number of divisions the centroblast has already gone through. It is sufficient to numerically solve the problem for the one- and two-generation case in order to see why only the one-generation model is consistent with experimental data. While in this model centrocytes greatly outnumber centroblasts during the main part of the reaction [Fig. 3(a)] as observed by MacLennan and coworkers (MacLennan et al., 1990), this is not the case in the two-generation model [Figure 3(b)]. The situation is even worse for more generations. Thus, only the one-generation model is consistent with experimental observations.
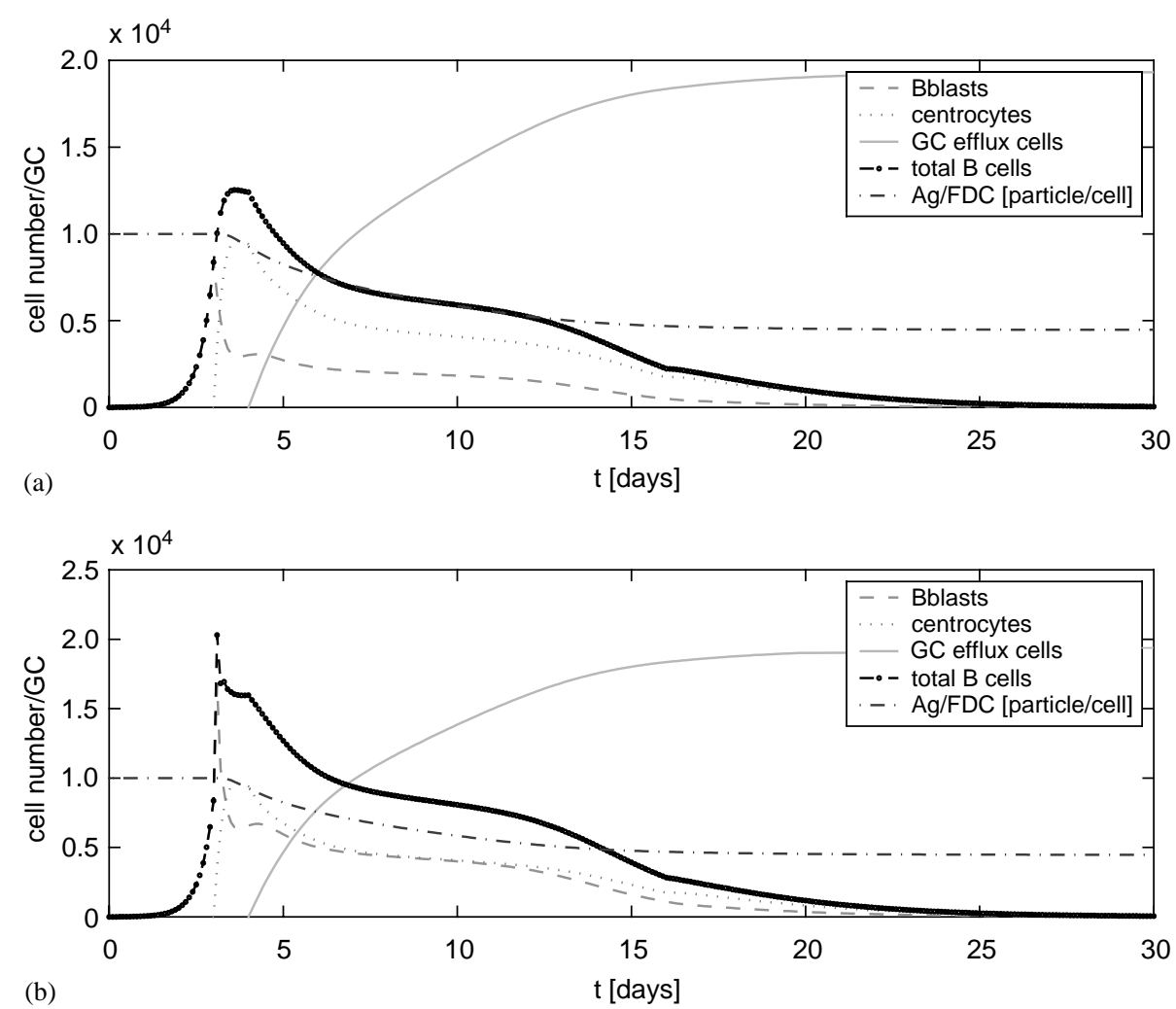

FIG. 3. GC kinetics of the primed primary immune response in the one-generation model where selection $(s)$ and recycling $\left(p_{r}\right)$ is dependent on antigen density, and somatic hypermutation terminates at day 16. (a) One-generation model: The dynamics of all cell populations behave as described in Fig. 2. (b) Two-generation model: The dynamics of all cell populations behave as described in Fig. 2 except that the centroblast and the centrocyte population are of similar size. 
EXTENSION OF THE BASIC MODEL TO INCLUDE AFFINITY MATURATION

From the simple model for the GC reaction we now know that centroblasts probably differentiate into centrocytes after one cell division. With respect to the timing of somatic hypermutation we have seen from experiments that a termination at about day 16 still allows the model to reproduce GC kinetics. We now turn to modelling affinity maturation and in this context we will investigate possible benefits of terminating somatic hypermutation well before the end of the $\mathrm{GC}$ reaction.

Given that in the experimental work on affinity maturation on which we base this model only low-middle- and high-affinity clones are distinguished (Smith et al., 1997), it is sensible at this stage to restrict ourselves to two species, namely high- and low-affinity clones. We keep track of the cell populations in these affinity classes by using vectors of size two where the first argument refers to the number of cells with low affinity of the given cell species and the second argument to the number of cells with high affinity. The two components are denoted by subscripts. Mutation is modelled by applying a mutation matrix $\mathbf{M}$ and $m$ is therefore replaced by $m \mathbf{M}$ in eqn (2). We study and compare two scenarios, one with somatic hypermutation termination at about day 16 and one without such termination. During times when somatic hypermutation is active we have $m=0.72$ and $\mathbf{M}$ as given in eqn (34). For all other times we have $m=1$ and $\mathbf{M}$ is the unit matrix. The constants differ for high- and low-affinity clones and are therefore written as pairs of numbers where the first argument refers to the constant for lowaffinity clones and the second argument to the constant for high-affinity clones. In explicit form the system which we are going to solve looks as follows:

For $t \leqslant 3$

$$
\frac{\mathrm{d} B}{\mathrm{~d} t}=\rho B
$$

For $t>3$

$$
\frac{\mathrm{d} B_{1}}{\mathrm{~d} t}=p_{r_{1}} \rho C_{S_{1}}-\rho B_{1},
$$

$$
\frac{\mathrm{d} B_{2}}{\mathrm{~d} t}=p_{r_{2}} \rho C_{S_{2}}-\rho B_{2},
$$

$$
\begin{gathered}
\frac{\mathrm{d} C_{1}}{\mathrm{~d} t}=2 \rho m\left(\mathbf{M}_{11} B_{1}+\mathbf{M}_{12} B_{2}\right)-\mu_{1} C_{1}, \\
\frac{\mathrm{d} C_{2}}{\mathrm{~d} t}=2 \rho m\left(\mathbf{M}_{21} B_{1}+\mathbf{M}_{22} B_{2}\right)-\mu_{2} C_{2} \\
\frac{\mathrm{d} C_{S_{1}}}{\mathrm{~d} t}=d s_{1} C_{1}-\rho C_{S_{1}}, \\
\frac{\mathrm{d} C_{S_{2}}}{\mathrm{~d} t}=d s_{2} C_{2}-\rho C_{S_{2}}, \\
\frac{d M_{1}}{\mathrm{~d} t}=\left(1-p_{r_{1}}\right) C_{S_{1}}, \\
\frac{d M_{2}}{\mathrm{~d} t}=\left(1-p_{r_{2}}\right) C_{s_{2}}
\end{gathered}
$$

with $\mu_{i}=d s_{i}+\delta_{c}\left(1-s_{i}\right)$.

The biological relevance of the different entries of $\mathbf{M}$ is as follows:

$\mathbf{M}_{11}$ - proportion of low-affinity cells that remain low affinity;

$\mathbf{M}_{12}$ - proportion of high-affinity cells that become low affinity;

$\mathbf{M}_{21}$ - proportion of low-affinity cells that become high affinity;

$\mathbf{M}_{22}$ - proportion of high-affinity cells that remain high affinity.

Given that the total number of cells does not change, the proportions need to sum to 1 , so $\mathbf{M}_{11}+\mathbf{M}_{21}=1$ and $\mathbf{M}_{12}+\mathbf{M}_{22}=1$.

Affinity bins and mutation matrices have been used before in models of affinity maturation (Kepler \& Perelson, 1993) although somatic hypermutation is believed to be a somewhat random process. The existence of hot spots (Neuberger et al., 1998) and the large number of cells justify a deterministic modelling approach. The use of affinity bins can be justified by the limited resolution of measurements. 


\section{ANALYSIS OF THE AFFINITY MATURATION MODEL}

Stability Analysis and the Possible

Parameter Range of the Selection Factor $s$ and the Recycling Probability $p_{r}$

In order to reproduce the experimental results the solution needs to tend to the trivial equilibrium point after day 4. The set of parameters for which this is achieved was determined by considering the quasistatic case when the selection rate $s$ and the recycling probability $p_{r}$ are constant and then by using the Routh-Hurwitz criterion. The possible parameter range is dependent on the proportion of beneficial mutations as can easily be seen in Fig. 4. Here, for four different choices of $\mathbf{M}_{11}$, those parameters, for which the trivial solution is not the stable steady state, are interconnected leaving the possible parameter space white. The larger the $\mathbf{M}_{11}$ the lesser the restrictions lie on the parameters for high-affinity clones (the selection probability $s_{2}$ and the recycling probability $p_{r 2}$ ). This is also intuitively understandable since for large $\mathbf{M}_{11}$ only few high-affinity clones are generated in every round of mutation and the population reducing effects therefore have to preferentially act on the low-affinity population in order to prohibit the GC population from growing. We further see that high recycling rates are compatible with stable or falling population size if only a small proportion of all clones becomes selected as has already been observed in experiments.

\section{Efficiency of Affinity Maturation}

Given that the main focus of this paper is the process of affinity maturation, it is now of interest to determine the restrictions on the parameters such that we indeed obtain affinity maturation.
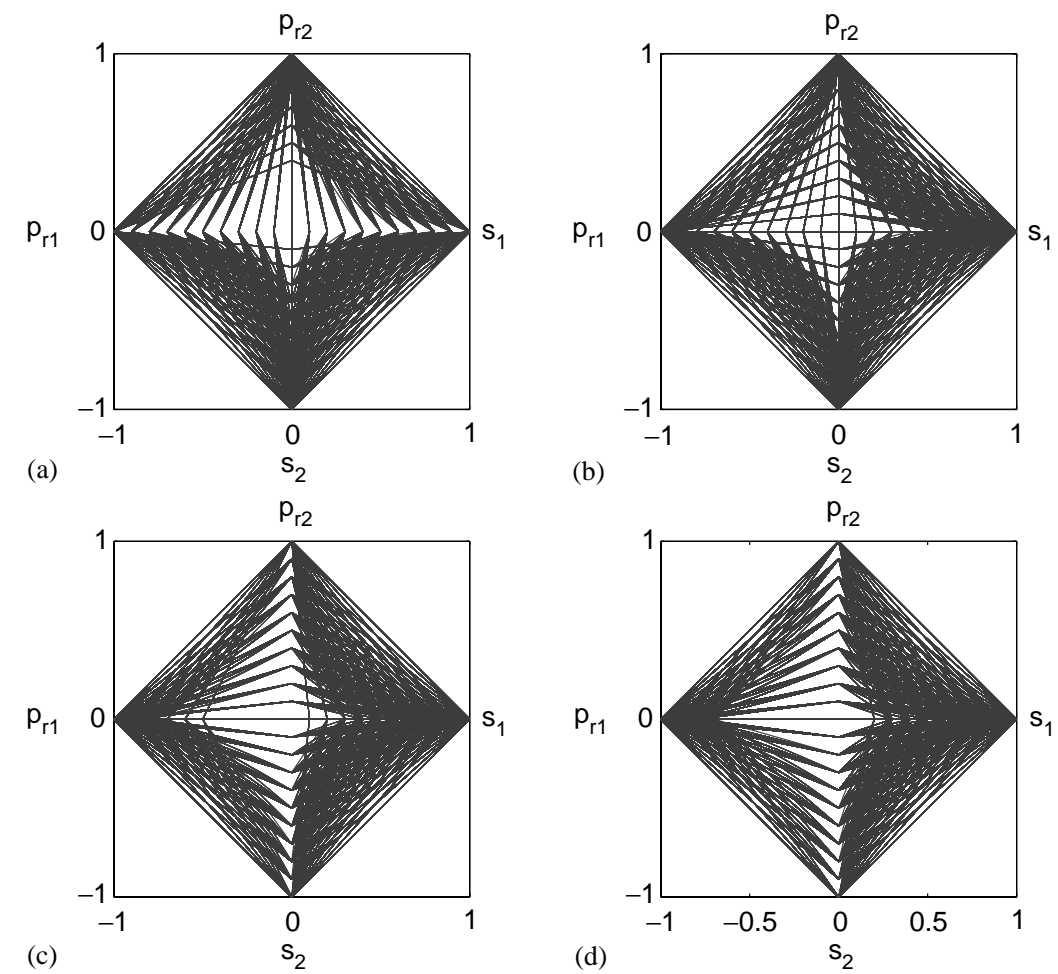

FIG. 4. Parameter region for which the system cannot attain the trivial equilibrium point: (a) $\mathbf{M}_{11}=0.65$; (b) $\mathbf{M}_{11}=$ 0.75 ; (c) $\mathbf{M}_{11}=0.85$; (d) $\mathbf{M}_{11}=0.95$. The parameter range for $s_{1}$ is depicted on the positive $x$-axis, the parameter range for $p_{r 1}$ on the negative $x$-axis, while $s_{2}$ and $p_{r 2}$ are represented on the negative and positive $y$-axis, respectively. Parameter sets for which the trivial equilibrium point cannot be attained are connected by lines. For increasing $\mathbf{M}_{11}$ the restriction on the relevant parameters of the low-affinity population $\left(s_{1}\right.$ and $\left.p_{r 1}\right)$ increases while the restriction on the same parameters for the high-affinity population decreases. 
We can analyze the efficiency of affinity or maturation best by defining

$$
\begin{aligned}
& \dot{T}_{1}=\dot{B}_{1}+\dot{C}_{1}+\dot{C}_{s 1}+\dot{M}_{1}, \\
& \dot{T}_{2}=\dot{B}_{2}+\dot{C}_{2}+\dot{C}_{s 2}+\dot{M}_{2}
\end{aligned}
$$

so that $\dot{T}_{1}\left(\dot{T}_{2}\right)$ represents the rate of increase of low (high)-affinity cells. We therefore have a net affinity increase if $\dot{T}_{1}<\dot{T}_{2}$. This inequality is satisfied if the following conditions hold:

$$
\begin{gathered}
C_{s 1}<C_{s 2} \frac{1-p_{r 2}}{1-p_{r 1}}, \\
C_{1}>C_{2} \frac{1-s_{2}}{1-s_{1}}, \\
\frac{B_{1}}{B_{2}}<\frac{-1+2 m\left(\mathbf{M}_{22}-\mathbf{M}_{12}\right)}{-1+2 m\left(\mathbf{M}_{11}-\mathbf{M}_{21}\right)} .
\end{gathered}
$$

From inequality (27) we see that affinity maturation is favoured if high-affinity clones recycle with less probability than low-affinity clones $\left(p_{r 2}<p_{r 1}\right)$. We will come back to this idea when we analyze the mutation matrix in more detail. Inequality (28) yields the intuitively understandable insight that it is most beneficial for affinity maturation if low-affinity clones are selected to a lesser degree than high-affinity clones $\left(s_{1}<s_{2}\right)$.

Inequality (29) can be further analyzed by using $\quad \mathbf{M}_{11}+\mathbf{M}_{21}=1, \quad \mathbf{M}_{12}+\mathbf{M}_{22}=1 \quad$ and $\mathbf{M}_{21}+\mathbf{M}_{12}=\beta$ where $\beta$ is the proportion of mutations that lead to an affinity change. We obtain from eqn (29) the condition

$$
\frac{B_{1}}{B_{2}}<\frac{-1+2 m\left(3-2 \beta-2 \mathbf{M}_{11}\right)}{-1+2 m\left(2 \mathbf{M}_{11}-1\right)} .
$$

Given that the LHS of inequality (29) is bounded below by 0 , we require the RHS to be larger than zero in order to obtain affinity maturation. This is achieved if either

$$
\begin{aligned}
& \mathbf{M}_{11}>0.5+0.25 m^{-1} \text { and } \\
& \beta<1.5-0.25 m^{-1}-\mathbf{M}_{11}
\end{aligned}
$$

$$
\begin{aligned}
& \mathbf{M}_{11}<0.5+0.25 m^{-1} \text { and } \\
& \beta>1.5-0.25 m^{-1}-\mathbf{M}_{11}
\end{aligned}
$$

Estimates by others yield $\beta=0.52$ and $m=$ 0.72 (Shannon \& Mehr, 1999) and condition (31) can therefore not be satisfied, while condition (32) implies $\mathbf{M}_{11}<0.85$. Note that since inequalities (27)-(29) are sufficient but not necessary, the upper limit for $\mathbf{M}_{11}$ may in fact be higher. The RHS of inequality (30) goes to zero when $\mathbf{M}_{11}$ approaches its upper limit and for large $\mathbf{M}_{11}$ we can therefore only obtain affinity maturation if the LHS, $B_{1} / B_{2}$, can be made very small, which can be achieved by stringent selection conditions on the low-affinity population.

\section{A Multi-Generation Mechanism Cannot be Expected to Lead to a Higher Degree of Affinity Maturation}

After we have ruled out a multi-generation mechanism for centroblast-centrocyte conversion for kinetic reasons, we can now make use of the formalism developed above to also show that such a mechanism cannot be expected to be of great value with respect to affinity maturation. Introduction of several centroblast generations leads to a multiple application of the matrix $\mathbf{M}$ on the centroblast vector while condition (29) is changed to

$$
\begin{array}{r}
\frac{\sum_{i} B_{1}^{i}}{\sum_{j} B_{2}^{j}}<\frac{-1+2 m\left(\mathbf{M}_{22}-\mathbf{M}_{12}\right)}{-1+2 m\left(\mathbf{M}_{11}-\mathbf{M}_{21}\right)} \\
=\frac{-1+2 m\left(3-2 \beta-2 \mathbf{M}_{11}\right)}{-1+2 m\left(2 \mathbf{M}_{11}-1\right)}
\end{array}
$$

for $i, j=1, \ldots, n$ being the number of divisions the centroblasts have gone through. While the RHS is identical to the case for the onegeneration model, the LHS of inequality (33) now includes the sum of all centroblast populations.

It is not difficult to show that if inequality (29) is satisfied there is a net shift of the mutation matrix $\mathbf{M}$ to high affinity (i.e. $\mathbf{M}_{22}>\mathbf{M}_{11}$ ) if the population of low-affinity centroblasts is larger than the population of high-affinity centroblasts 
by more than $\mathbf{M}_{12} / \mathbf{M}_{21}$. Multiplication of the mutation matrix with itself, however, does not change the ratio $\mathbf{M}_{12}^{n} / \mathbf{M}_{21}^{n}$ as can be seen from the explicit calculation of $\mathbf{M}^{n}$ with $n=2$ :

$$
\mathbf{M}^{2}=\left(\begin{array}{cc}
\mathbf{M}_{11}^{2}+\mathbf{M}_{12} \mathbf{M}_{21} & \mathbf{M}_{12}\left(\mathbf{M}_{11}+\mathbf{M}_{22}\right) \\
\mathbf{M}_{21}\left(\mathbf{M}_{11}+\mathbf{M}_{22}\right) & \mathbf{M}_{22}^{2}+\mathbf{M}_{12} \mathbf{M}_{21}
\end{array}\right) .
$$

From this we see that the ratio between worsening and improving mutations is constant if the matrix is multiplied by itself. This implies that if the population of low-affinity clones is larger than the population of high-affinity clones by more than $\mathbf{M}_{12} / \mathbf{M}_{21}$, then we have a net increase in the high-affinity population upon application of this matrix. If, however, the proportion of high-affinity clones is higher, then this matrix lowers this proportion. In the limit we can therefore only attain the ratio $\mathbf{M}_{12} / \mathbf{M}_{21}$ by consecutive application of this matrix. This is illustrated in Fig. 5, and we further see that the increase in the proportion of high-affinity clones per application of the mutation matrix is highest when the proportion of high-affinity clones is smallest and if $n$, the number of times the mutation matrix has already been applied, is small. This result is therefore also not in favor of a multi-generation model.

\section{Termination of Somatic Hypermutation Before the end of the GC Reaction is Beneficial for Affinity Maturation}

In case of a termination of somatic hypermutation $\mathbf{M}$ becomes the unit matrix and inequality (29) reduces to the inequality $B_{1} / B_{2}<1$. This condition can be easily satisfied by introducing more stringent selection conditions which reduce the number of $B_{1}$ cells. The $B_{2}$ cells further multiply and soon dominate the germinal centre reaction. From this it is obvious that it is beneficial to terminate somatic hypermutation before the germinal centre reaction finishes in

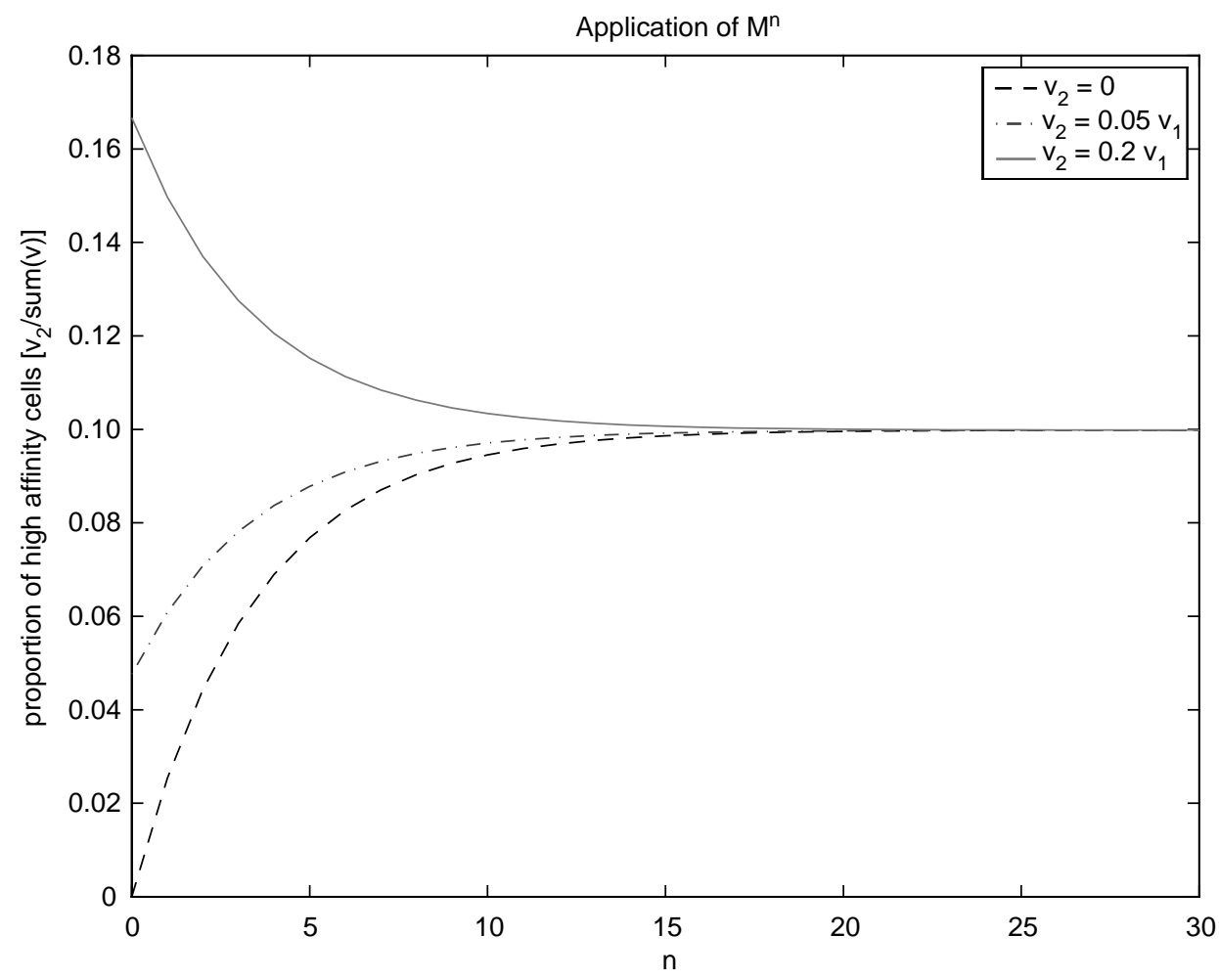

FIG. 5. Consecutive application of the mutation matrix M. The proportion of high-affinity cells $v_{2} / \sum_{i} v_{i}$ is plotted against the number of times $n$ the mutation matrix $\mathbf{M}$ was applied. The shift per application of the mutation matrix depends on the initial proportion of high-affinity cells, $v_{2}$, given in the legend in the inset. 
order to be able to further expand the highaffinity clones without the risk of losing them because of worsening mutations. It is also clear that somatic hypermutation must not terminate before high-affinity clones have evolved. Given that high-affinity clones have about nine mutations (Siekevitz et al., 1987; Wedemayer et al., 1997), and assuming that one way through the cycle takes about a day, we need about 9 days of somatic hypermutation. Given that not every mutation is beneficial the 12 days allowed for somatic hypermutation to take place seems to be reasonable.

\section{NUMERICAL SOLUTION OF THE FULL MODEL}

After having gained insights concerning the number of centroblast generations, the optimal time span of somatic hypermutation, the optimal choice of the selection factor and the recycling probability, the final step to take is to solve numerically the full model of the GC reaction including affinity maturation. Modelling affinity maturation allows us to finally include the competition between centrocytes and emerging high-affinity antibodies as a driving force of affinity maturation. The resulting model is robust against variations in the initial antigen amount over several orders of magnitude and against variations in the proportion of beneficial mutations.

First, biologically reasonable values need to be deduced for all parameters. For most rates the range can be justified while the correct value remains elusive and would require further experiments. However, the quality of the estimates should be sufficient for the predictions made to be of biological interest. In order to find the correct entries for $\mathbf{M}$ we use calculations by others (Shannon \& Mehr, 1999) that estimate $53 \%$ of all mutations to be silent and $28 \%$ to be lethal. Therefore, from the surviving $72 \%, 74 \%$ have not changed affinity while $26 \%$ have. We cannot expect that more than $10 \%$ of all mutations which change the affinity (or about $2 \%$ of all mutations) are beneficial. Manser even assumes that only $0.1 \%$ of all mutations are beneficial (Manser, 1990), a figure which may, however, be quite pessimistic. Assuming that $10 \%$ of all changing mutations are beneficial we obtain as mutation matrix

$$
\mathbf{M}=\left(\begin{array}{ll}
0.948 & 0.468 \\
0.052 & 0.532
\end{array}\right)
$$

which is derived as follows. If $10 \%$ of all changing mutations were beneficial, 2.6\% of all low-affinity clones would be promoted to high affinity in every round of mutation. We therefore have $\mathbf{M}_{21}=0.1 \times 0.26 \times 2=0.052$ where the factor 2 comes in since the sum over all entries of $M$ is 2. The remaining part of the low-affinity clones remains of low-affinity and we therefore find $\mathrm{M}_{11}=1-0.052=0.948$. Given that we assume that $26 \%$ of all mutations change the affinity $(26-2.6) \%=23.4 \%$ must reduce the affinity so that $\mathbf{M}_{12}=0.234 \times 2=$ 0.468 . The remaining high-affinity clones remain of high-affinity and we find for the last matrix entry $\mathbf{M}_{22}=1-0.468=0.532$.

Given that the division rate is $\rho=\frac{24}{6.5} \mathrm{day}^{-1}$ and we apply the mutation matrix continuously in every time step, we need to take $\mathbf{M}$ to the power $\left[24 \mathrm{day}^{-1} / 6.5\right] \times \mathrm{d} t$ with $\mathrm{d} t=0.1$ being our numerical finite time difference. Therefore, the actual value we take for the matrix $\mathbf{M}$ is

$$
\mathbf{M}=\left(\begin{array}{ll}
0.9763 & 0.2136 \\
0.0237 & 0.7864
\end{array}\right) .
$$

These mutations change the proportion between high- and low-affinity clones, which is calculated from

$$
\mathscr{A}=\frac{B_{2}+C_{2}+C_{s 2}+M_{2}}{\sum_{i}\left(B_{i}+C_{i}+C_{s i}+M_{i}\right)} .
$$

Recall, that when we investigated the antigen dependency on the selection factor $s$ and the recycling probability $p_{r}$ in the previous section, we had to use unrealistically high values for the Hill coefficient $n$, and the basic model of the GC reaction was not robust to changes in the initial number of antigen complexes (a failing of the model since the amount of deposited antigen varies between GC reactions (MacLennan, pers. comm. 2001) and robustness of the real GC reaction to variations of several orders of magnitude has been observed). The GC reaction could attain 
such stability by a feedback mechanism such that in case of a high availability of antigen the total cell number would be reduced. A possible candidate for such a feedback mechanism is antigen masking by high-affinity antibodies $(A b)$. On the other hand, antibodies with an affinity marginally above average may still be out-competed by centrocytes. The emergence of high-affinity antibodies in the serum has been determined experimentally for a true primary immune response (Smith et al., 1994; 1997). The emergence of antibodies follows a saturation law and the largest increase in antibody concentration was found between day 7 (when high-affinity antibodies can first be found) and day 14. A simplistic way of modelling the kinetics of high-affinity antibody emergence is given by

$$
\begin{aligned}
& \frac{\mathrm{d} A b}{\mathrm{~d} t}=\kappa A F C_{2}-k_{\text {on }} A g A b \\
& \text { with } \kappa=10^{8} \frac{\text { antibody }}{\text { day } \times \text { cell }} \\
& k_{\text {on }}=5 \times 10^{-13} \text { day }^{-1} \text { particle }^{-1}
\end{aligned}
$$

which is derived as follows. Given that high-affinity antibodies are produced by highaffinity plasma cells $\left(A F C_{2}\right)$ it is sensible to assume that the increase in antibody concentration is proportional to the size of this cell population (first summand in eqn (36)) while the decrease is proportional to antibody:antigen complex formation (second summand in eqn (36)). The on-rate of antibody: antigen binding, $k_{\text {on }}=5 \times 10^{-13}$ day $^{-1}$ particle $^{-1}$ $\simeq 3.5 \times 10^{6} \mathrm{M}^{-1} \mathrm{~s}^{-1}$ was chosen in the range of what has been measured for high-affinity antibodies in experiments (Batista \& Neuberger, 1998; Neri et al., 1995) (see Table 2). A maximal value for $\kappa$, the production rate of high-affinity antibodies that are retained within the GC, cannot be expected to be smaller than $10^{11}$ antibodies per cell per day. However, it is reasonable to assume that the major part of all antibodies produced is either already produced outside of GCs or is at least not retained within the GC. Given that we consider high-affinity interactions, antibody:antigen binding is considered to be irreversible on the time-scale considered and $k_{\text {off }}$ is set to be zero. Natural decay of antibody by catabolizing processes as well as by other parts of the immune response such as the innate are neglected. The stoichiometry of the antigen:antibody complex depends on the antigen. Given that there is no data available on the amount of antibody found in GCs it is, however, not sensible to worry about stoichiometries at this stage. With this model we can only investigate whether antigen masking by antibody could drive affinity maturation efficiently-further experiments are then needed to elucidate this point.

The availability of free, unbound antigen will decrease due to extraction as discussed before and due to antigen masking and we therefore have

$$
\begin{aligned}
& \frac{\mathrm{d} A g}{\mathrm{~d} t}=-\left(u k C+u C_{s}(1-k)\right) A g-k_{o n} A g A b \\
& \text { with } u=10^{-4} \frac{\text { particle }}{\text { cell } \times \text { day }} .
\end{aligned}
$$

The on-rate $k_{\text {on }}=5 \times 10^{-13} \mathrm{day}^{-1}$ particle $^{-1}$ $\simeq 3.5 \times 10^{6} \mathrm{M}^{-1} \mathrm{~s}^{-1}$ is used as before. $u$, the extraction rate of antigen by centrocytes, is again chosen such that only about half of the antigen vanishes from the germinal centre. Due to the GC reaction, most of the remaining antigen will be present in the form of antigen:antibody complexes, $K_{A g A b}$, whose emergence is modelled by

$$
\begin{aligned}
\frac{\mathrm{d} K_{A g A b}}{\mathrm{~d} t} & =k_{o n} A g A b \quad \text { with } \\
k_{\text {on }} & =5 \times 10^{-13} \text { day }^{-1} \text { particle }^{-1} .
\end{aligned}
$$

Our choice of the selection factor $s$ and the recycling probability $p_{r}$ is guided by the analysis presented above. In order to gain maximal affinity increase we need to choose $s_{1}$ small while $s_{2}$ has to be large. The recycling probability, on the other hand, needs to be chosen high for low-affinity clones $\left(p_{r 1}\right)$ and low for high-affinity clones $\left(p_{r 2}\right)$. The recycling probability for high-affinity clones has to increase with time, reflecting the stronger competition for activating signals because of reduction 
of available antigen. We therefore have

$$
\begin{gathered}
\mathbf{s}=\left(\begin{array}{c}
0.01+0.09 \zeta \\
0.1+0.85 \zeta
\end{array}\right), \\
\mathbf{p}_{\mathbf{r}}=\left(\begin{array}{c}
0.95 \\
0.7 \eta
\end{array}\right)
\end{gathered}
$$

with $\zeta=A g / \sum_{i} C_{i}$ and $\eta=\min \left(K_{A g A b} / \sum_{i} C_{i}, 1\right)$, where $\zeta$ models the fact that less antigen per centrocyte leads to less activation of B cells even if their BCRs recognize the antigen with high affinity. $\eta$ accounts for the negative feedback of antigen:antibody complexes on B cell signalling which is here assumed to cause more recycling and less $\mathrm{B}$ cell differentiation to plasma and memory cells.

In the scenario where termination of somatic hypermutation is included, it occurs simultaneously with increased selection stringency. Both processes may therefore be regulated by the same mechanism and we assume that somatic hypermutation terminates when $\zeta<1$. A more realistic case would be a continuous decrease of somatic hypermutation with decreasing $\zeta$.

The centrocytes which do not recycle can either differentiate into memory or antibodyproducing plasma cells (also called antibodyforming cells (AFCs)). It has been observed that early selected centrocytes preferentially leave the GC reaction and differentiate into antibodyforming cells, many of which migrate to the bone marrow where they persist for several weeks and secrete antibodies (Smith et al., 1997). The proportion of high-affinity clones among AFCs located in the spleen during the measurements has been determined explicitly in the experiments and we will use these data to validate the model.

There is inconclusive data concerning memory cells $(M)$. While from histological investigations it was claimed that memory cells can first be found between days 3 and 4 (MacLennan, pers. comm., 2001), investigations of the antibody repertoire support the concept that memory cells develop late during the response. Theoretical analysis shows that the affinity of the memory cell pool is higher, the later memory cell formation starts (Meyer-Hermann et al., 2001) although at least in the model presented here the difference caused by a late start is not very high. In vitro experiments have shown that there may exist a feedback loop which inhibits plasma cell formation once there is a high titre of $\mathrm{IgG}$ antibodies (Tarlinton \& Smith, 2001). In the true primary immune response they can be detected in the serum at day 7 (Smith et al., 1994; 1997). Taken together the dynamics of plasma and memory cells can be modelled by

$$
\begin{gathered}
\frac{\mathrm{d} A F C}{\mathrm{~d} t}=\theta \rho\left(1-p_{r}\right) C_{s}, \\
\frac{\mathrm{d} M}{\mathrm{~d} t}=(1-\theta) \rho\left(1-p_{r}\right) C_{s},
\end{gathered}
$$

where $\theta=0.3\left(A g / \sum_{i} C_{i}\right)\left(A g /\left(A g+K_{A g A b}\right)\right)$ is the product of the ratio of free antigen to centrocytes and the fraction of free antigen, and models the inhibition of AFC formation by less availability of free antigen and by the emergence of IgG:antigen complexes.

Figures 6 and 7 show numerical simulation results for the cases without termination of somatic hypermutation or with termination, respectively. In both cases, the overall kinetics behave as before and as described in the literature (Liu et al., 1991). Given that we are still considering a one-generation model, centrocytes still outnumber centroblasts as observed by MacLennan and co-workers (MacLennan et al., 1990). As found in experiments (Tew \& Mandels, 1979), the total amount of antigen is only halved during the immune response (curve for $\mathrm{Ag}: \mathrm{Ab}$ complexes in Figs 6 and 7), while the amount of free antigen is reduced to zero due to masking by soluble high-affinity antibodies which are produced by plasma cells. This masking drives the competition in the germinal centres and leads to affinity maturation (Figs. 6 and 7 middle panel) as observed in experiments (Smith et al., 1997). The total amount of free antibody in the GCs obtained in this simulation corresponds to a concentration of about $1 \mathrm{M}$ which is reasonable since we have not considered the fact that most of the antibodies will be produced outside the GC or diffuse out, and in experiments millimolar concentrations of antibody are already sufficient to block binding. The GC reaction and the affinity maturation in 

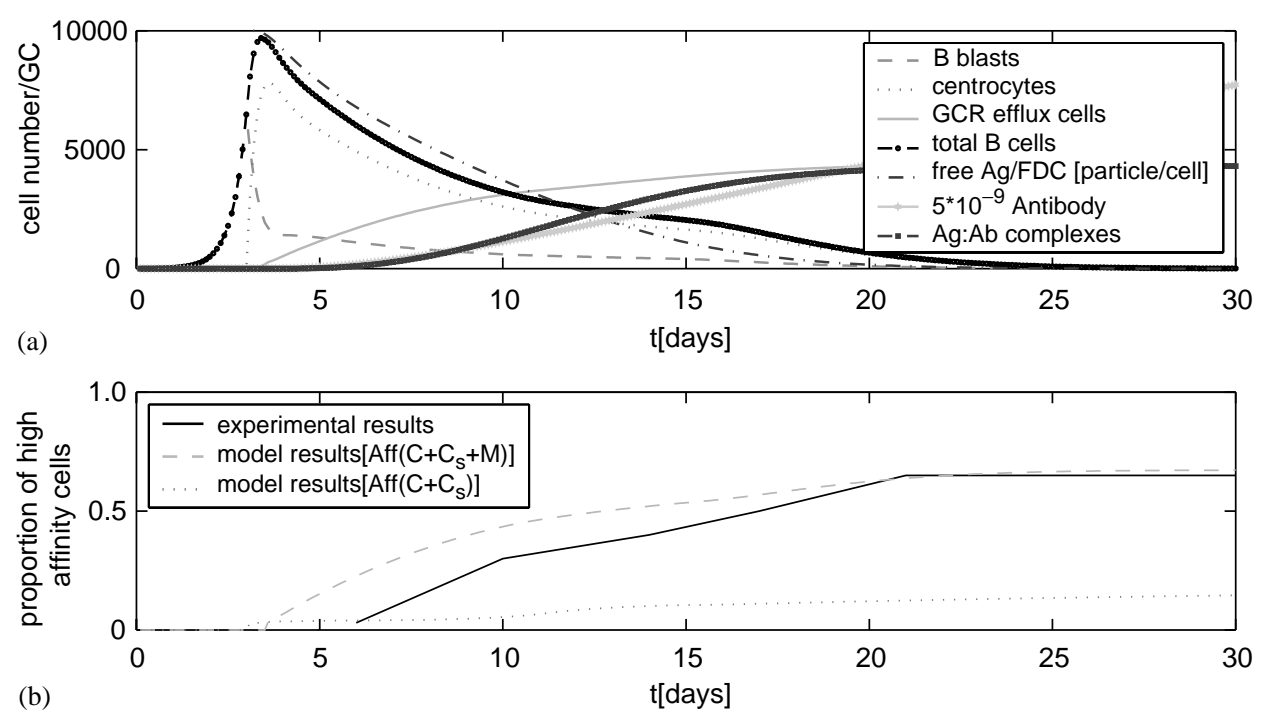

(b)

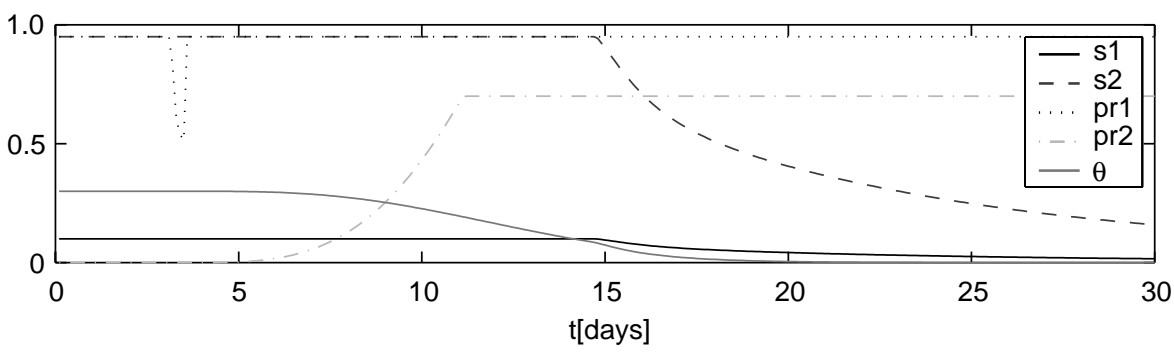

FIG. 6. GC kinetics and affinity maturation of the primed primary immune response without termination of somatic hypermutation. (a) GC kinetics of the primed primary immune response in the one-generation model. The dynamics of all cell populations behave as described before. (b) Affinity maturation during the primed primary immune response: The solid line represents experimental results for a true primary immune response (Smith et al., 1997) which starts later than the primed primary immune response. The model results for the affinity increase in centrocyte (c), selected centrocyte $\left(C_{S}\right)$ and memory cell $(M)$ population together (broken line) are close to those observed experimentally. The earlier onset of affinity maturation seen in the model results from the nature of the primed primary immune response. The dotted line represents the affinity increase in the whole centrocyte population $\left(C+C_{s}\right)$. (c) The dynamic behaviour of the selection and recycling probability for low- and high-affinity clones is depicted as well as $\theta$, the fraction of free antigen.

the model are very stable against increases in the initial amount of antigen over several orders of magnitude. This is in good agreement with the observation that the amount of deposited antigen varies (MacLennan, pers. comm., 2001) and experiments in which the antigen amount was strongly increased but the reaction was found to be normal (Vora et al., 1997). Also the proportion of beneficial mutations can be varied over a wide range without affecting the GC reaction or affinity maturation substantially. This robustness is very important since the proportion of beneficial mutations will strongly depend on the antigen encountered. Also in favour of both models is the proportion of high-affinity AFCs at day 14. Although nothing was specifically undertaken to influence this number, the proportion given in the literature $(56 \%)$ (Smith et al., 1997) is reproduced well by the model $(65.5 \%)$. The antibody level increases exponentially in this model while a saturation curve was obtained in the experiments (Smith et al., 1997). The reason for this is that in this model no further antibody decreasing processes such as natural decay and the immune response against the invader are considered.

The only major difference between the simulations with and without termination of somatic hypermutation is the extent of affinity maturation. As predicted before, termination of somatic hypermutation leads to the expansion of high-affinity clones which finally dominate the 

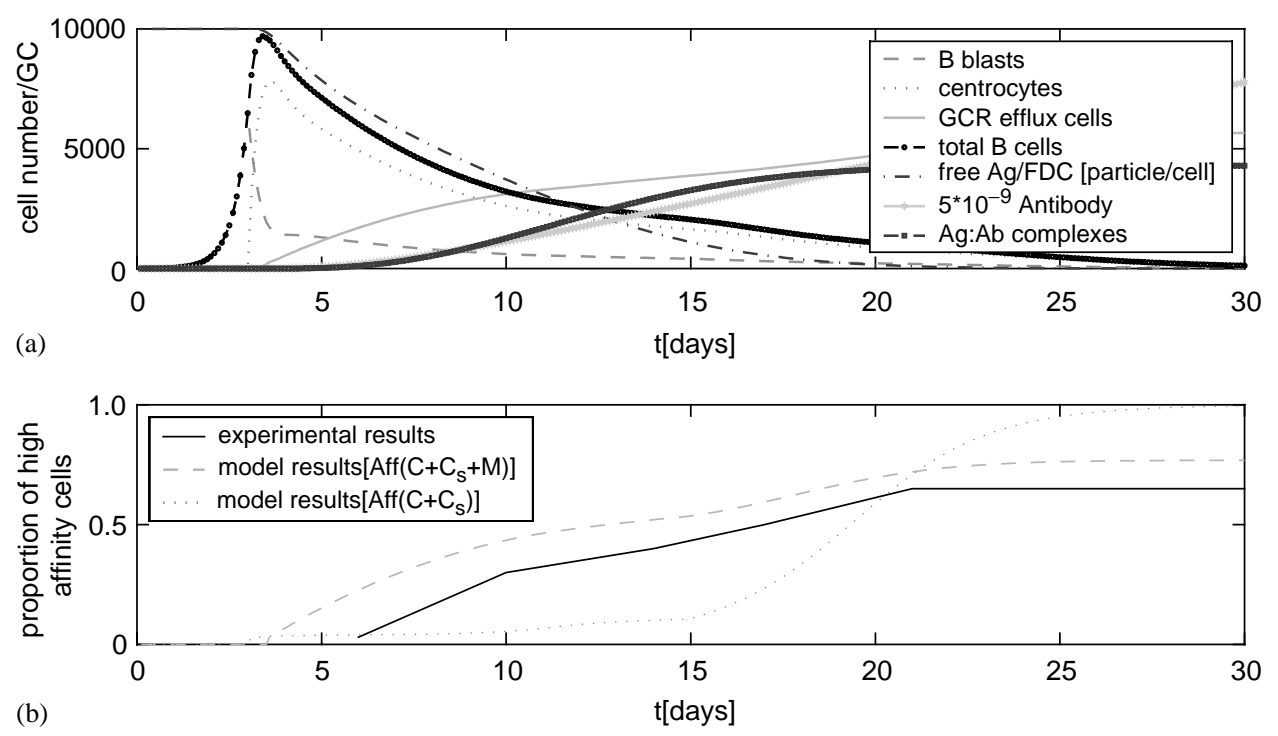

(b)

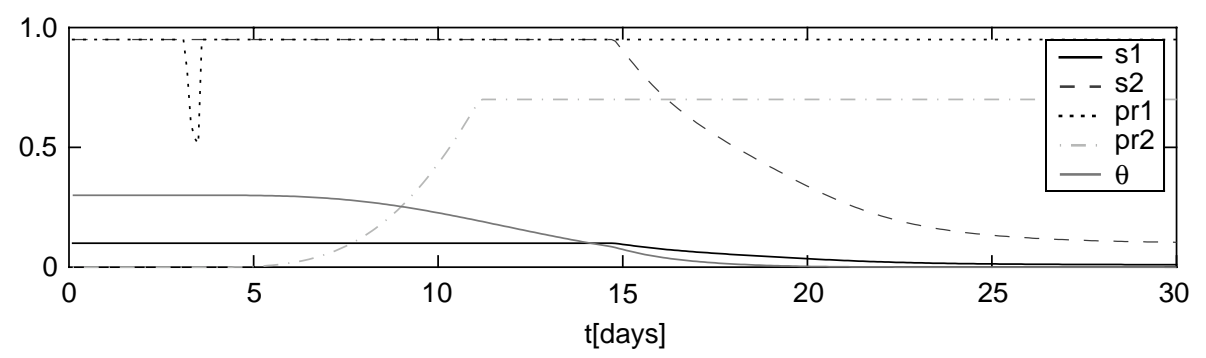

FIG. 7. GC kinetics and affinity maturation of the primed primary immune response if somatic hypermutation terminates before the end of the GC reaction. (a) GC kinetics of the primed primary immune response in the one-generation model. The dynamics of all cell populations behave as described before. (b) Affinity maturation during the primed primary immune response if somatic hypermutation terminates before the end of the GC reaction. The solid line represents experimental results for a true primary immune response (Smith et al., 1997) which starts later than the primed primary immune response. The model results for the affinity increase in centrocyte (c), selected centrocyte $\left(C_{S}\right)$ and memory cell $(M)$ population together (broken line) are close to those observed experimentally except for the final affinity increase after day 16. Until about day 16 the affinity increase is largely due to an affinity increase in the memory population. After the termination of somatic hypermutation the average affinity increases again substantially due to an affinity increase in the centrocyte population $\left(C+C_{s}\right)$ which is represented by the dotted line. Termination of somatic hypermutation before the end of the GC reaction is therefore beneficial for affinity maturation and would be necessary to reproduce experimental data if a lower proportion of improving mutations had been chosen. The earlier onset of affinity maturation seen in the model results from the nature of the primed primary immune response. (c) The dynamic behaviour of the selection and recycling probability for low- and high-affinity clones is depicted as well as $\theta$, the fraction of free antigen.

response (Fig. 7 middle panel). This is a further point in favour of termination of somatic hypermutation at about day 16 as has been suggested before by several experimentalists (Berek \& Milstein, 1988; Jacob et al., 1993). One further way to check the model is to impair recycling $\left(p_{r}=0\right)$. As has been shown experimentally (de Vinuesa et al., 2000) this leads to a break-down of the GC reaction (see Fig. 8).

The last step is to build a multi-bin model. However, given that neither entries for the much more complex mutation matrix nor values for the different entries of $s$ or $p_{r}$ are known, it is sensible to wait for more experimental data to be available before undertaking a detailed modelling approach.

\section{Discussion}

\section{KINETIC ASPECTS}

(a) Regulation of centroblast to centrocyte differentiation: No experiments have been carried out to address the regulation mechanism for the differentiation of centroblasts into 


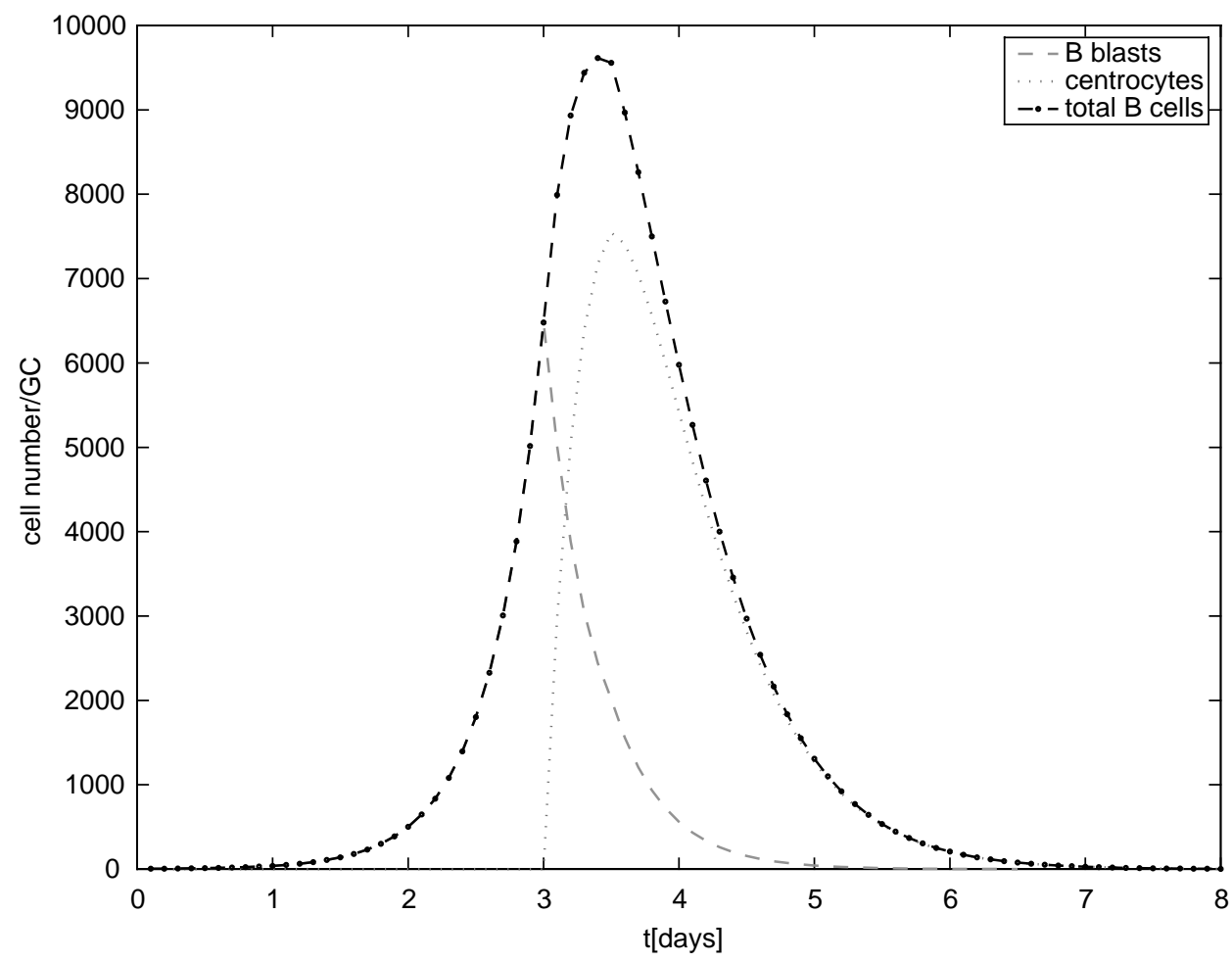

FIG. 8. GC kinetics during the primed primary immune response if recycling is impaired as observed in experiments (de Vinuesa et al., 2000): the GC reaction breaks down between day 5 and 6 of the response.

centrocytes. From the kinetic data available we can rule out both a cell-density-dependent and a time-dependent mechanism. From the observation of MacLennan and co-workers (MacLennan et al., 1990) that centrocytes greatly outnumber centroblasts during the main part of the GC reaction it also follows that centroblasts differentiate into centrocytes after one cell division since otherwise both cell populations are either of the same size or centroblasts even outnumber centrocytes (see Fig. 7). This result implies a much simpler mechanism for conversion than the model of Kesmir and de Boer (1999) where it was proposed that centroblasts do not differentiate before having gone through at least four divisions, raising the question as to how centroblasts sense through how many generations they have already gone and whether somatic hypermutation acts on each generation.

(b) Start of memory cell formation: Recently, a model has been proposed in which a non-output phase is shown to be beneficial for affinity maturation (Meyer-Hermann et al., 2001). This leaves the question as to how the initiation of memory cell formation is regulated. If $\operatorname{IgG}$ at least partially inhibits AFC formation as measured in vitro (Ridderstad \& Tarlinton, 1998; Tarlinton \& Smith, 2000), memory cell formation may increase with time as was assumed in this model. Such a regulation would allow the body to produce antibodies as a first defence against rapidly dividing pathogens until the titre of high-affinity $\mathrm{IgG}$ antibodies is sufficiently high and a feedback inhibition of AFC formation leads to a switch to more memory cell formation. AFCs and memory cells are also found in the bone marrow and other places in the body. It is, however, unknown which proportion emigrates and whether and how often cells divide before leaving the GC. Because of this lack of data emigration was not incorporated in the model.

There is a long-running debate on whether or not there is a further selection step for cells to either enter the memory or plasma cell pool (Smith et al., 1997). There are certainly signals which bias selected centrocytes to differentiate in either direction (Liu et al., 1997) but given that 
the model presented here reproduces the experimental proportions of high-affinity clones without considering such a further selection step, properties other than affinity may be important for differentiation in either direction.

\section{THE EFFICIENCY OF AFFINITY MATURATION}

(a) The role of selection and recycling: The process of the immune response would be most efficient if somatic hypermutation worked on a small number of centroblast generations $(n<10)$ and if the initial and recycling centroblast population had a very small proportion of high-affinity clones. This has indeed been shown for the initial centroblast population which was found to consist of 3\% high-affinity clones (Smith et al., 1997). In order to keep the proportion of high-affinity centroblasts low, the recycling probability for high-affinity centroblasts needs to be much smaller than for lowaffinity clones. Such a regime would also be compatible with inequality (27) as discussed above. This is a new insight into the importance of this parameter for affinity maturation and raises the question of how high-affinity clones could be generated from low-affinity clones if clones of improved affinity hardly recycle and high-affinity clones cannot be generated from low-affinity clones in one round. This conceptual problem arises because of the simplicity of our model which only considers two different affinities so that a shift from low- to high-affinity is possible in this model.

At this stage it is therefore beneficial to consider an advanced model with 10 bins. The number 10 is sensible since experimentalists find that high-affinity B cells differ in their antibody coding genes by about eight mutations from germline genes. Assuming that every one of these mutations changes the affinity of the antibody, it is sensible to use eight bins for improved affinity, one for the starting affinity and one for reduced/no affinity. While cells below the starting bin undergo apoptosis when trying to interact with antigen, cells well above the starting bin are selected into the plasma cell pool or into the memory cell pool. The cells in the bins in between mostly recycle, some of them may also enter the memory pool. The threshold above which cells definitely leave the GC as memory or plasma cells can be assumed to increase during the germinal centre reaction. This is in agreement with the analysis of the two-bin model: once most of the population has gone, say from bin 2 to bin 3 , bin 2 acts as the low-affinity bin in our model and is assigned the recycling probability for low-affinity cells instead of that of high-affinity cells. Therefore, the recycling probability changes with time for the different bins and finally even clones of relatively high-affinity recycle and are expanded. In this model, high-affinity clones preferentially leave the GC reaction at the beginning. This is also intuitively understandable since at the beginning a high-affinity clone, which was generated by chance, would be lost with high probability once it goes through mutation again since most of the mutations worsen the antibody. Additionally, the body needs early high-affinity clones in order to hold the invader in check. Early emigration is therefore beneficial for the efficiency of affinity maturation and for the early immune response. Once, however, many clones have reached the next affinity bin and mutation acts on a wide platform, mutations are beneficial since they will not lead to a complete elimination of the already gained beneficial mutations but there is a good chance that affinity is even enhanced.

The early emigration of high-affinity clones has been described previously (Radmacher et al., 1998; Smith et al., 1997) but was understood to be due to a stochasticity in the selection and was viewed as a failure of the system (Radmacher et al., 1998). The model presented here suggests that the early emigration is a deterministic process and is beneficial for affinity maturation and for the defence of the organism against the invader since the early emigrants produce antibodies which support the organism's first defence. This finding is also in good agreement with another experimental result where a transgene which coded for a high-affinity antibody was inserted into the genome of an animal (Vora \& Manser, 1995). The animal nevertheless produced a similar memory cell repertoire as the control animals without the transgene. This favours the notion of deterministic early 
emigration of high-affinity clones which therefore do not dominate the response. In the later response animals without the transgene have obtained antibodies of comparable affinity by mutation while the antibody encoded by the transgene can accumulate worsening mutations.

The model also explains the results of Hannum and co-workers (Hannum et al., 2000) which show that the germinal centre reaction is more or less normal even if the animals cannot deposit antigen on FDCs due to the lack of soluble antibodies. Their data show that the GC cell numbers are even higher than in the control animals while the activation of centrocytes is reduced. Due to less activation, presumably more cells recycle and the GC reaction is therefore stable. The activation may be lower since antigen cannot be displayed professionally on FDCs but needs to be recognized in other (maybe even in soluble) form which elicits lower response (Batista et al., 2001).

The selection factor needs to be chosen in the opposite way to the recycling rate and is high for high-affinity clones and low for low-affinity clones. This is also intuitively understandable. With time competition for antigen increases because extraction of antigen by centrocytes lowers the antigen density. Therefore, the selection proportion of low affinity clones decreases with time.

(b) The role of termination of somatic hypermutation: The analysis of this model shows that termination of somatic hypermutation before the end of the germinal centre reaction is beneficial for affinity maturation since it allows the expansion of high-affinity clones without the hazard of losing the clone due to worsening mutations. The signal for a switch-off of somatic hypermutation may be less activation due to less availability of free antigen since this would also provide a mechanism for an increase in the selection stringency at the same time. The correlation may be due to a connection between activating signals during centrocyte selection and signals for continuation of somatic hypermutation after centrocytes have recycled. The signal may also be received directly by centroblasts which have been shown recently to express surface immunoglobulin in the late stages of the GC (Camacho et al., 1998). During that stage the dark and light zones can no longer be seen and the FDC network extends through the whole germinal centre such that centroblasts can directly interact with FDCs. With the beginning of the second week of the response, these FDCs have further been shown to express CD23, also known as FceRII (Camacho et al., 1998). CD23 has been shown to bind to CD21, which modulates signalling through BCR. Time dynamic expression of various such surface molecules may regulate somatic hypermutation termination.

In total, we conclude that antigen masking by evolving antibody is an effective way by which affinity maturation may be driven. The postulated feedback effect on AFC formation may further regulate the extent to which $\mathrm{B}$ cells either leave the $\mathrm{GC}$ or recycle. Understanding the mechanism and regulation of recycling may be key to understanding affinity maturation as from this model the extent to which B cells leave or recycle has a very strong impact on affinity maturation. This is also sensible from a biological point of view since selection is mainly meant to have a protective function against the emergence of self-reactive clones. It is not sensible to eliminate even weak binders which can be used as plasma cells as a first defence against the invader. Furthermore, the model strongly supports the concept of an early termination of somatic hypermutation at about day 16 since without such a termination, affinity maturation would be less efficient as termination of somatic hypermutation allows the expansion of the high-affinity B cell population without the hazard of worsening mutations.

This model made use of several black boxes in which less well characterized steps were included. Before refining the model, predictions made by the model must be tested experimentally, and more data obtained on the dependence of B cell selection on BCR:antigen affinity and antigen density. Furthermore, it would be interesting to have data on the impact of antigen masking by antibody on affinity maturation and on fate determination by the quality of BCR:antigen and B cell:T cell interaction. Finally, it would be helpful if the sizes of the different $B$ cell populations (centroblasts, centrocytes, memory cells, plasma cells) were known in detail. 
DI would like to thank D. Gavaghan and M.C. MacLennan for useful discussions and acknowledges support from an EPSRC scholarship and the Studienstiftung des deutschen Volkes. This work was completed while PKM was a Visiting Senior Research Fellow at the Isaac Newton Institute for Mathematical Sciences, University of Cambridge.

\section{REFERENCES}

Allen, D., Cumano, A., Dildrop, R., Kocks, C., Rajewsky, K., Rajewsky, N., Roes, J., Sablitzky, F. \& Siekevitz, M. (1987). Timing, genetic requirements and functional consequences of somatic hypermutation during B cell development. Immunol. Rev. 96, 5-22.

Batista, F. D. \& Neuberger, M. S. (1998). Affinity dependences of the $\mathrm{B}$ cell response to antigen: a threshold, a ceiling and the importance of off-rate. Immunity $\mathbf{8}$, 751-759.

Batista, F. D., Iber, D. \& Neuberger, M. S. (2001). B cells acquire antigen from target cells after synapse formation. Nature 411, 489-494.

Batista, F. D. \& Neuberger, M. (2000). B cells extract and present immobilized antigen: implications for affinity discrimination. EMBO J. 19, 513-520.

Berek, C. \& Milstein, C. (1988). The dynamic nature of the antibody repertoire. Immunol. Rev. 105, 5-26.

Berek, C., Berger, A. \& Apel, M. (1991). Maturation of the immune response in germinal centers. Cell 67, 1121-1129.

Camacho, S. A., Kosco-Vilbois, M. H. \& Berek, C. (1998). The dynamic structure of the germinal center. Immunol. Today 19, 511-514.

De Vinuesa, C. G., Cook, M. C., Ball, J., Drew, M., Sunners, Y., Cascalho, M., Wabl, M., Klaus, G. G. B. \& MacLennan, I. C. (2000). Germinal centers without T cells. J. Exp. Med. 191, 485-493.

Goodnow, C. C. G. (1997). Chance encounters and organized rendezvous. Immunol. Rev. 156, 5-10.

Han, S., Zheng, B., DalPorto, J. \& Kelsoe, G. (1995). In situ studies of the primary immune response to (4-hydroxy-3-nitrophenyl)acetyl. iv. Affinity-dependent, antigen-driven B cell apoptosis in germinal centers as a mechanism for maintaining self-tolerance. J. Exp. Med. 182, 1635-1644.

Hannum, L. G., Haberman, A. M., Anderson, S. M. \& Shlomchiк, M. J. (2000). Germinal center initiation, variable gene region hypermutation, and mutant $\mathrm{B}$ cell selection without detectable immune complexes on follicular dendritic cells. J. Exp. Med. 192, 931-942.

Hardie, D. L., Johnson, G. D., Khan, M. \& MacLenNAN, I. C. (1993). Quantitative analysis of molecules which distinguish functional compartments within germinal centers. Eur. J. Immunol. 23, 997-1004.

JaCOB, J., Kassir, R. \& Kelsoe, G. (1991). In situ studies of the primary immune response to (4-hydroxy-3nitrophenyl)acetyl. I. the architecture and dynamics of responding cell populations. J. Exp. Med. 173, $1165-1175$.

Jacob, J., Kelsoe, G., Rajewsky, K. \& Weiss, U. (1991). Intraclonal generation of antibody mutants in germinal centres. Nature 354, 389-392.
Jacob, J., Przylepa, J., Miller, C. \& Kelsoe, G. (1993). In situ studies of the primary immune response to (4-hydroxy-3-nitrophenyl)acetyl. III. The kinetics of V region mutation and selection in germinal center B cells. J. Exp. Med. 178, 1293-1307.

Janeway, C. A. \& Travers, P. (1997). Immunology. The Immune System in Health and Disease. New York: Garland Publisher.

Kepler, T. B. \& Perelson, A. S. (1993). Somatic hypermutation in B cells: an optimal control treatment. J. theor. Biol. 164, 37-64.

Kesmir, C. \& De Boer, R. J. (1999). A mathematical model on germinal center kinetics and termination. J. Immunol. 163, 2463-2469.

Kroese, F. G. M., Wubena, A. S., Seijen, H. G. \& Nieuwenhuis, P. (1987). Germinal centers develop oligoclonally. Eur. J. Immunol. 17, 1069-1072.

Kuppers, R., ZhaO, M., Hansmann, M.-L. \& Rajewsky, K. (1993). Tracing B cell development in human germinal centres by molecular analysis of single cells picked from histological sections. EMBO J. 12, 4955-4967.

Lanzavecchia, A. (1985). Antigen-specific interaction between T and B cells. Nature 314, 537-539.

Liu, Y. L., Joshua, D. E., Smith, C. A. G. J. \& MacLennan, I. C. (1989). Mechanisms of antigen-driven selection in germinal centres. Nature 342, 929-931.

LiU, Y. L., Zhang, J., Chan, E. Y. T. \& MacLennan, I. C. (1991). Sites of specific B cell activation in primary and secondary responses to T-cell-dependent and T-cell-independent antigens. Eur. J. Immunol. 21, 2951-2962.

Liu, Y. J., De Bouteiller, O. \& Fugier-Vivier, I. (1997). Mechanisms of selection and differentiation in germinal centers. Curr. Opin. Immunol. 9, 256-262.

Maclennan, I. C. (1994). Germinal centers. Annu. Rev. Immunol. 12, 117-139.

Maclennan, I. C., LiU, Y. L., Oldfield, S., Zhang, J. \& LANe, P. J. L. (1990). The evolution of B-cell clones. Curr. Toppers Microbiol. Immunol. 159, 37-64.

Maclennan, I. C., Garciá De Vinuesa, C. \& CasaMAYOR-PALleJA, M. (2000). B-cell memory and the persistence of antibody responses. Philos. Trans. R. Soc. London B Biol. Sci. 355, 345-350.

MAnser, T. (1990). The efficiency of antibody affinity maturation: can the rate of B-cell division be limiting? Immunol. Today 11, 305-308.

Meyer-Hermann, M., Deutsch, A. \& Or-Guil, M. (2001). Recycling probability and dynamical properties of germinal centre reactions. J. theor. Biol. 210, 265-285.

Neri, D., Momo, M., Prospero, T. \& Winter, G. (1995). High-affinity antigen binding by chelating recombinant antibodies (crabs). J. Mol. Biol. 246, 367-373.

Neuberger, M. S., Ehrenstein, M. R., Klix, N., Jolly, C. J., Yelamos, J., Rada, C. \& Milstein, C. (1998). Monitoring and interpreting the intrinsic features of somatic hypermutation. Immunol. Rev. 162, 107-116.

Oprea, M. \& Perelson, A. S. (1997). Somatic mutation leads to efficient affinity maturation when centrocytes recycle back to centroblasts. J. Immunol. 158, 5155-5162.

Radmacher, M. D., Kelsoe, G. \& Kepler, T. B. (1998). Predicted and inferred waiting times for key mutants in the germinal centre reaction: evidence for stochasticity in selection. Immunol. Cell Biol. 76, 373-381. 
Ridderstad, A. \& Tarlinton, D. M. (1998). Kinetics of establishing the memory B cell population as revealed by CD38 expression. J. Immunol., 160, 4688-4695.

Shannon, M. \& Mehr, R. (1999). Reconciling repertoire shift with affinity maturation: the role of deleterious mutations. J. Immunol. 162, 3950-3956.

Siekevitz, M., Kocks, C., Rajewsky, K. \& Dildrop, R. (1987). Analysis of somatic mutations and class switching in naive and memory $\mathrm{B}$ cells generating adoptive primary and secondary responses. Cell 48, 757-770.

Smith, K. G. C., Weiss, U., Rajewski, K., Nossal, G. J. V. \& TARLINTON, D. M. (1994). Bcl-2 increases memory B cell recruitment but does not perturb selection in the germinal centers. Immunity 1, 803-813.

Smith, K. G. C., Light, A., Nossal, G. J. V. \& TARlinton, D. M. (1997). The extent of affinity maturation differs between the memory and antibodyforming cell compartments in the primary immune response. EMBO J. 16, 2996-3006.

Stryer, L. (1995). Biochemistry. New York: W.H. Freeman and Company.
Tarlinton, D. M. \& Smith, K. G. C. (2000). Dissecting affinity maturation: a model explaining selection of antibody-forming cells and memory $\mathrm{B}$ cells in the germinal centre. Immunol. Today 9, 436-441.

Tew, J. G. \& Mandel, T. E. (1979). Prolonged antigen half-life in the lymphoid follicles of specifically immunized mice. Immunology 37, 69-76.

Vora, K. A. \& Manser, T. (1995). Altering the antibody repertoire via transgene homologous recombination: evidence for global and clone-autonomous regulation of antigen-driven B cell differentiation. J. Exp. Med. 181, 271-281.

Vora, K. A., Ravetch, J. V. \& Manser, T. (1997). Amplified follicular immune complex deposition in mice lacking the $\mathrm{Fc}$ receptor $\gamma$-chain does not alter maturation of the $\mathrm{B}$ cell response. J. Immunol. 159, 2116-2124.

Wedemayer, G. J., Patten, P. A., Wang, L. H., Schultz, P. G. \& Stevens, R. C. (1997). Structural insights into the evolution of an antibody combining site. Science 276, 1665-1669. 